\title{
Minimal element theorems revisited
}

\author{
Andreas H. Hamel, Constantin Zălinescu ${ }^{\dagger}$
}

July 16, 2021

\begin{abstract}
Starting with the Brezis-Browder principle, we give stronger versions of many variational principles and minimal element theorems which appeared in the recent literature. Relationships among the elements of different sets of assumptions are discussed and clarified, i.e., assumptions to the metric structure of the underlying space and boundedness assumptions. New results involving set-valued maps and the increasingly popular set relations are obtained along the way.
\end{abstract}

Keywords. variational principle, minimal element theorem, set-valued map, set relation, locally convex space

MSC2010. Primary 46N10 Secondary 58E30, 49J53

\section{Introduction}

The celebrated variational principle due to Ekeland ensures the existence of minimal elements with respect to (wrt for short) an order relation on a complete metric space $(X, d)$ generated by a lower semicontinuous function $f: X \rightarrow \mathbb{R} \cup\{+\infty\}$ which is bounded from below; this order is defined by

$$
x_{1} \preceq x_{2} \quad: \Longleftrightarrow f\left(x_{1}\right)+d\left(x_{1}, x_{2}\right) \leq f\left(x_{2}\right) .
$$

The assumptions required from $f$ lead to certain features of $\preceq$ whereas the assumptions to $X$ admit a countable induction argument which produces a Cauchy sequence which is decreasing wrt $\preceq$ and whose limit then is the desired minimal element.

Quite some effort went into attempts to generalize this result.

In the first line of research, the assumptions to $X$, a (complete) metric space, are basically kept, but the order relation (11) is replaced by a more general one. A blueprint result of this type is [3, Theorem 3.2], others include [30, Theorem 1], 9, Theorem 16] (for this, see also [13, Theorem 2.2]) and [19, Lemma 2.2]. Such results can also be applied to order relations of the type (11) with $f$ a vector- or even set-valued function which makes them very powerful tools. The thesis [9] exemplifies this approach with corollaries for functions $f$ mapping in preordered monoids (of sets), for example. Below, Corollaries 3.9, 3.10, 3.11, 3.12 are of this type. One could also look at these results as attempts to separate the countable induction argument already used in the proof of [4, Theorem 1] from the features of the order relation;

\footnotetext{
${ }^{*}$ Free University of Bozen-Bolzano, Faculty of Economics and Management, University Square 1, 39031 Bruneck-Brunico, Italy

†University "Al. I. Cuza" Iasi, Faculty of Mathematics, Bd. Carol I, 11, 700506 Iasi, Romania
} 
applications consist in a mere check if the order relation in question satisfies the required assumptions which links it to the metric structure. Therefore, the mentioned results can be proven without involving the Brezis-Browder principle as shown in the alternative proof of Theorem 3.7 below.

The second line of research is concerned with order relations on arbitrary sets (without a metric structure, for example). Of course, alternative requirements have to be added. A prominent result of this type is the Brezis-Browder principle [1] (BB-principle) where the existence of a real-valued function is assumed which is bounded from below and increasing wrt the order relation. Further examples for such results can be found in [25, 27] and also in [31. Of course, BB-type theorems can also be used to obtain the results on metric spaces discussed in the previous paragraph with a suitable monotone function; the proof of the BB-principle also involves a countable induction argument (see the first proof of Theorem 3.2 below).

A third goal is to lift the results from order relations on a set $X$ to such relations on a product set $X \times Z$. Results in this direction have been obtained first by Göpfert and Tammer (see [5. Section 3.10] and the references 140-144 therein) and are usually called minimal element theorems.

In this note, all three of the above lines are followed providing very general results and discussing the relationships between different sets of assumptions in detail. Consequently, most relevant results in the literature are obtained as special cases. In particular, it is shown that variational principles and minimal element theorems involving (very general) set relations can be obtained (see, for example, Corollary 3.14 below). Such results can be found in [8] for complete metric spaces (manuscript version [7] from 2002) and more general ones in [12] (preprint version [11] from 2002), the latter reference already giving a proof based on the BB-principle and nonlinear scalarization.

\section{Preliminary Notions and Results}

Let $\mathbb{N}$ denote the set of natural numbers including 0 and $\mathbb{R}$ the real numbers. The set $\mathbb{R}_{+}$is the set of all nonnegative real numbers.

In the sequel $(X, d)$ is a metric space, $Y$ is a real separated topological vector space, $Y^{*}$ is its topological dual, and $K \subseteq Y$ is a proper convex cone; as usual, $K^{+}$is the positive dual cone of $K$ and $K^{\#}$ is the quasi-interior of $K^{+}$:

$$
\begin{aligned}
& K^{+}=\left\{y^{*} \in Y^{*} \mid y^{*}(y) \geq 0 \forall y \in K\right\}, \\
& K^{\#}=\left\{y^{*} \in Y^{*} \mid y^{*}(y)>0 \forall y \in K \backslash\{0\}\right\},
\end{aligned}
$$

where for $\alpha, \alpha^{\prime} \in \mathbb{R}, \alpha \leq \alpha^{\prime}$ (or, equivalently, $\alpha^{\prime} \geq \alpha$ ) means, as usual, that $\alpha^{\prime}-\alpha \in \mathbb{R}_{+}$, while $\alpha<\alpha^{\prime}$ (or $\alpha^{\prime}>\alpha$ ) means that $\alpha^{\prime}-\alpha \in \mathbb{R}_{+} \backslash\{0\}$.

If $Y$ is just a real linear space we (can) endow it with the finest locally convex topology, that is, the core convex topology (see [14, Exercise 2.10]).

The preorder $\leq_{K}$ on $Y$ generated by $K$ via

$$
y_{1} \leq_{K} y_{2} \quad: \Longleftrightarrow y_{2} \in y_{1}+K
$$

for $y_{1}, y_{2} \in Y$ can be extended to the power set $2^{Y}$ by

$$
A_{1} \leq_{K}^{l} A_{2} \quad: \Longleftrightarrow \quad A_{2} \subseteq A_{1}+K,
$$


and it is easily seen that $\leq_{K}^{l}$ is reflexive, transitive, thus a preorder, but not a partial order in general even if $\leq_{K}$ is antisymmetric. See [10] for more details and references. Here and in the following, the addition for sets is understood in the Minkowski (element-wise) sense with the convention $\emptyset+A=A+\emptyset=\emptyset$ for all $A \in 2^{Y}$. It can also be checked that $\leq_{K}^{l}$ is compatible with this addition as well as with multiplication by non-negative numbers.

As in [29] and [15], let $F: X \times X \rightrightarrows K$ satisfy the conditions:

(F1)

$$
0 \in F(x, x) \text { for all } x \in X \text {, }
$$

$$
F\left(x_{1}, x_{2}\right)+F\left(x_{2}, x_{3}\right) \subseteq F\left(x_{1}, x_{3}\right)+K \text { for all } x_{1}, x_{2}, x_{3} \in X .
$$

Of course, (F2) is the triangle inequality for $F$ wrt $\leq_{K}^{l}: F\left(x_{1}, x_{3}\right) \leq_{K}^{l} F\left(x_{1}, x_{2}\right)+F\left(x_{2}, x_{3}\right)$; moreover, when $K$ is pointed, $(\mathrm{F} 1)$ is $F(x, x) \leq_{K}^{l}\{0\}$ since $F$ maps into $K$.

Remark 2.1 Obviously,

$$
A_{1} \leq_{K}^{l} A_{2} \quad \Longleftrightarrow \quad A_{2}+K \subseteq A_{1}+K
$$

since $0 \in K$. The collection of sets $A \subseteq Y$ satisfying $A=A+K$ is denoted by $\mathcal{P}(Y, K)$, and $\leq_{K}^{l}$ coincides with $\supseteq$ on $\mathcal{P}(Y, K)$.

Therefore, in many cases (but not all), one can replace $F$ by the function $F_{K}: X \times X \rightrightarrows K$ defined by $F_{K}\left(x_{1}, x_{2}\right)=F\left(x_{1}, x_{2}\right)+K$. Indeed, if $F$ satisfies (F1) and (F2), then $F_{K}$ does as well. In this case, $F_{K}(x, x)=K$ for all $x \in X$, and $F_{K}$ is an order premetric in the sense of $[9$, Def. 30] mapping into the preordered monoid $\left(\mathcal{P}(Y, K),+, \leq_{K}^{l}\right)$ with the Minkowski addition.

For $F$ satisfying conditions $(\mathrm{F} 1)$ and $(\mathrm{F} 2)$, and $z^{*} \in K^{+}$, consider

$$
\eta_{F, z^{*}}: X \times X \rightarrow \overline{\mathbb{R}}_{+}, \quad \eta_{F, z^{*}}\left(x, x^{\prime}\right):=\inf \left\{z^{*}(z) \mid z \in F\left(x, x^{\prime}\right)\right\} .
$$

It follows immediately that

$$
\eta_{F, z^{*}}(x, x)=0 \text { and } \eta_{F, z^{*}}\left(x, x^{\prime \prime}\right) \leq \eta_{F, z^{*}}\left(x, x^{\prime}\right)+\eta_{F, z^{*}}\left(x^{\prime}, x^{\prime \prime}\right) \quad \forall x, x^{\prime}, x^{\prime \prime} \in X .
$$

Using $F$ we introduce a preorder on $X \times 2^{Y}$, denoted by $\preceq_{F}$, in the following manner:

$$
\left(x_{1}, A_{1}\right) \preceq_{F}\left(x_{2}, A_{2}\right): \Longleftrightarrow A_{1}+F\left(x_{1}, x_{2}\right) \leq_{K}^{l} A_{2} \Longleftrightarrow A_{2} \subset A_{1}+F\left(x_{1}, x_{2}\right)+K ;
$$

clearly, $A_{2}=\emptyset \Rightarrow\left(x_{1}, A_{1}\right) \preceq_{F}\left(x_{2}, A_{2}\right)$, and $\left[\left(x_{1}, A_{1}\right) \preceq_{F}\left(x_{2}, A_{2}\right), A_{2} \neq \emptyset\right] \Rightarrow\left[A_{1} \neq \emptyset\right.$, $\left.F\left(x_{1}, x_{2}\right) \neq \emptyset\right]$. Indeed, $\preceq_{F}$ is reflexive by (F1). Assume that $\left(x_{1}, A_{1}\right) \preceq_{F}\left(x_{2}, A_{2}\right)$ and $\left(x_{2}, A_{2}\right) \preceq_{F}\left(x_{3}, A_{3}\right)$. This means $A_{1}+F\left(x_{1}, x_{2}\right) \leq_{K}^{l} A_{2}$ and $A_{2}+F\left(x_{2}, x_{3}\right) \leq_{K}^{l} A_{3}$. Adding these two inequalities, applying (F2) and the transitivity of $\leq_{K}^{l}$ yields $A_{1}+F\left(x_{1}, x_{3}\right) \leq_{K}^{l} A_{3}$ which means that $\preceq_{F}$ is transitive.

Of course,

$$
\left(x_{1}, A_{1}\right) \preceq_{F}\left(x_{2}, A_{2}\right) \Longrightarrow A_{2} \subset A_{1}+K \Longleftrightarrow: A_{1} \leq_{K}^{l} A_{2} ;
$$

moreover, by (F1), we have that

$$
\left(x, A_{1}\right) \preceq_{F}\left(x, A_{2}\right) \Longleftrightarrow A_{2} \subset A_{1}+K \Longleftrightarrow A_{1} \leq_{K}^{l} A_{2} .
$$



by

Restricting $\preceq_{F}$ to $X \times\{A \mid A \subset Y$, card $A=1\}$ we get the preorder $\preceq_{F}^{1}$ on $X \times Y$ defined

$$
\left(x_{1}, y_{1}\right) \preceq_{F}^{1}\left(x_{2}, y_{2}\right) \quad: \Longleftrightarrow \quad y_{2} \in y_{1}+F\left(x_{1}, x_{2}\right)+K
$$

Hence, for $x, x_{1}, x_{2} \in X$ and $y_{1}, y_{2} \in Y$, we have that

$$
\begin{aligned}
\left(x_{1}, y_{1}\right) & \preceq_{F}^{1}\left(x_{2}, y_{2}\right) \Longleftrightarrow y_{1} \leq_{K} y_{2}, \\
\left(x, y_{1}\right) & \preceq_{F}^{1}\left(x, y_{2}\right) \Longleftrightarrow y_{1} \leq_{K} y_{2} .
\end{aligned}
$$

Notice that $\preceq_{F}^{1}$ is nothing else than $\preceq_{F}$ from [29].

Besides (F1) and (F2) we shall consider also the condition

(F3) there exists $z_{F}^{*} \in K^{+}$such that

$$
\eta(\delta):=\inf z_{F}^{*}\left(F_{\delta}\right):=\inf \left\{z_{F}^{*}(v) \mid v \in F_{\delta}\right\}>0 \quad \forall \delta>0,
$$

where

$$
F_{\delta}:=\cup\left\{F\left(x, x^{\prime}\right) \mid x, x^{\prime} \in X, d\left(x, x^{\prime}\right) \geq \delta\right\}
$$

for $\delta \geq 0$; it follows that $0 \leq \eta\left(\delta^{\prime}\right) \leq \eta(\delta)$ for $0 \leq \delta^{\prime}<\delta$ because $F_{\delta} \subseteq F_{\delta^{\prime}}$ in such a case.

Clearly, condition (F3) can be rewritten as

$$
\exists z_{F}^{*} \in K^{+}, \forall \delta>0: \inf z_{F}^{*}\left(F_{\delta}\right)>0 .
$$

A weaker condition is

$$
\forall \delta>0, \exists z^{*} \in K^{+}: \inf z^{*}\left(F_{\delta}\right)>0 .
$$

An even weaker condition is the following

$$
\forall \delta>0, \forall\left(z_{n}\right) \subseteq F_{\delta}, \exists z^{*} \in K^{+}: \lim \sup z^{*}\left(z_{n}\right)>0 ;
$$

when (13) holds Qiu [24, Def. 3.5] says that $F$ is compatible with $d$. Clearly, if (F3) holds then $0 \notin \overline{\operatorname{conv}} F\left(x, x^{\prime}\right)$ for $x \neq x^{\prime}$. Condition (F3) holds obviously if for some $z^{*} \in K^{+}$one has

$$
\forall x, x^{\prime} \in X: \inf _{z \in F\left(x, x^{\prime}\right)} z^{*}(z) \geq d\left(x, x^{\prime}\right) .
$$

The set below is related to the above conditions:

$$
K_{F}^{+}:=\left\{z^{*} \in K^{+} \mid z^{*}(z)>0 \forall z \in \cup_{\delta>0} F_{\delta}\right\} .
$$

In the sequel we consider a nonempty $K$-convex subset $H$ of $K$, that is $H+K$ is convex. It is worth observing that

$$
0 \leq \alpha \leq \beta \Rightarrow \beta H \subset \alpha H+K, \quad 0 \leq \gamma, \delta \Rightarrow \gamma H+\delta H \subset(\gamma+\delta) H+K
$$

The next result provides an important example of set-valued functions $F$ satisfying conditions (F1) and (F2) (see [15, Lem. 10.1.1]). 
Lemma 2.1 Let $\emptyset \neq H \subseteq K$ be a $K$-convex set. Consider

$$
F_{H}: X \times X \rightrightarrows K, \quad F_{H}\left(x, x^{\prime}\right):=d\left(x, x^{\prime}\right) H .
$$

Then

(i) $F_{H}$ verifies (F1) and (F2).

(ii) $F_{H}$ verifies condition (F3) iff $F_{H}$ verifies condition (12) iff there exists $z_{H}^{*} \in K^{+}$such that inf $z_{H}^{*}(H)>0$; if $Y$ is a separated locally convex space, then $F_{H}$ verifies condition (F3) iff $0 \notin \mathrm{cl}(H+K)$. Moreover, $F_{H}$ verifies condition (13) iff

$$
\forall\left(h_{n}\right) \subseteq H, \exists z^{*} \in K^{+}: \lim \sup z^{*}\left(h_{n}\right)>0 .
$$

Clearly, if $H$ is $K$-convex, then $H+K$ also is. The function $F_{H}+K=F_{H+K}$ satisfies (i) and (ii) of Lemma 2.1 and maps into $\mathcal{P}(Y, K)$.

Note that the case $0 \in H+K$ (that is $H \cap(-K) \neq \emptyset$ ) is not interesting in the present context because (the metric of) $X$ is not involved; indeed, in such a case $(x, A) \preceq_{F_{H}}\left(x^{\prime}, A^{\prime}\right)$ if and only if $A \leq_{K}^{l} A^{\prime}$. For this reason, in the sequel, $H$ is a nonempty $K$-convex subset of $K \backslash(-K)$. Moreover, we denote by $\preceq_{H}$ and $\preceq_{H}^{1}$ the preorders $\preceq_{F_{H}}$ and $\preceq_{F_{H}}^{1}$, respectively.

If (14) holds for $z^{*} \in K^{+}$, then

$$
\left(x_{1}, A_{1}\right) \preceq_{F}\left(x_{2}, A_{2}\right) \Longrightarrow \inf z^{*}\left(A_{1}\right)+d\left(x_{1}, x_{2}\right) \leq \inf z^{*}\left(A_{2}\right) .
$$

Indeed, from $\left(x_{1}, A_{1}\right) \preceq_{F}\left(x_{2}, A_{2}\right)$, we have that $A_{2} \subset A_{1}+F\left(x_{1}, x_{2}\right)+K$, and so $z^{*}\left(A_{2}\right) \subset$ $z^{*}\left(A_{1}\right)+z^{*}\left(F\left(x_{1}, x_{2}\right)\right)+z^{*}(K)$; it follows that

$$
\begin{aligned}
\inf z^{*}\left(A_{2}\right) & \geq \inf \left\{z^{*}\left(A_{1}\right)+z^{*}\left(F\left(x_{1}, x_{2}\right)\right)+z^{*}(K)\right\} \\
& =\inf z^{*}\left(A_{1}\right)+\inf z^{*}\left(F\left(x_{1}, x_{2}\right)\right)+\inf z^{*}(K) \\
& =\inf z^{*}\left(A_{1}\right)+\inf z^{*}\left(F\left(x_{1}, x_{2}\right)\right) \\
& \geq \inf z^{*}\left(A_{1}\right)+d\left(x_{1}, x_{2}\right)
\end{aligned}
$$

where, as usual, $\inf \emptyset:=+\infty$. Hence (19) holds. Using (19) for $A_{1} \subset Y$ with inf $z^{*}\left(A_{1}\right) \in \mathbb{R}$, we obtain that

$$
\left[\left(x_{1}, A_{1}\right) \preceq_{F}\left(x_{2}, A_{2}\right),\left(x_{2}, A_{2}\right) \preceq_{F}\left(x_{1}, A_{1}\right)\right] \Longrightarrow\left[x_{1}=x_{2}, \inf z^{*}\left(A_{1}\right)=z^{*}\left(A_{2}\right)\right] .
$$

In fact (21) holds if $F$ verifies conditions (F1)-(F3) and $A_{1} \subset Y$ is such that inf $z_{F}^{*}\left(A_{1}\right) \in \mathbb{R}$. Indeed for $\delta:=d\left(x_{1}, x_{2}\right)$, from (20) we get

$$
\inf z_{F}^{*}\left(A_{2}\right) \geq \inf z_{F}^{*}\left(A_{1}\right)+\inf z_{F}^{*}\left(F_{\delta}\right), \quad \inf z_{F}^{*}\left(A_{1}\right) \geq \inf z_{F}^{*}\left(A_{2}\right)+\inf z_{F}^{*}\left(F_{\delta}\right),
$$

and so $\inf z_{F}^{*}\left(A_{2}\right) \in \mathbb{R}$ and $-\left|\inf z_{F}^{*}\left(A_{2}\right)-\inf z_{F}^{*}\left(A_{1}\right)\right| \geq \inf z_{F}^{*}\left(F_{\delta}\right) \geq 0$.

Eq. (21) shows that $\preceq_{F}^{1}$ is antisymmetric (and so $\preceq_{F}^{1}$ is a partial order) when $F$ verifies conditions (F1)-(F3) with $z_{F}^{*} \in K^{\#}$. In what concerns $\preceq_{H}^{1}$ (for $H$ as above, that is $H \subset$ $K \backslash(-K)$ is nonempty and $K$-convex), we have the following:

$$
\left(x_{1}, y_{1}\right) \preceq_{H}^{1}\left(x_{2}, y_{2}\right) \preceq_{H}^{1}\left(x_{1}, y_{1}\right) \Longrightarrow x_{1}=x_{2} ;
$$

moreover, if $K$ is pointed, then $\preceq_{H}^{1}$ is antisymmetric. Indeed, take $\left(x_{1}, y_{1}\right) \preceq_{H}^{1}\left(x_{2}, y_{2}\right) \preceq_{H}^{1}$ $\left(x_{1}, y_{1}\right)$, and assume $\alpha:=d\left(x_{1}, x_{2}\right)>0$. Then $\pm\left(y_{1}-y_{2}\right) \in \alpha H+K=\alpha(H+K)$, and so 
$0 \in \alpha(H+K)$ by the convexity of $H+K$. Hence $0 \in H+K$, a contradiction. Therefore, (22) holds. Assume, moreover, that $K$ is pointed and $\left(x_{1}, y_{1}\right) \preceq_{H}^{1}\left(x_{2}, y_{2}\right) \preceq_{H}^{1}\left(x_{1}, y_{1}\right)$. Then, by (22) we have that $x_{1}=x_{2}$, and so $\pm\left(y_{1}-y_{2}\right) \in K$, whence $y_{1}=y_{2}$ because $K$ is pointed. Hence $\preceq_{H}^{1}$ is antisymmetric.

For $F$ satisfying conditions $(\mathrm{F} 1)$ and $(\mathrm{F} 2)$, and $z^{*} \in K^{+}$, we introduce the partial order $\preceq_{F, z^{*}}$ on $X \times 2^{Y}$ by

$$
\left(x_{1}, A_{1}\right) \preceq_{F, z^{*}}\left(x_{2}, A_{2}\right): \Longleftrightarrow\left\{\begin{array}{l}
\left(x_{1}, A_{1}\right)=\left(x_{2}, A_{2}\right) \text { or } \\
\left(x_{1}, A_{1}\right) \preceq_{F}\left(x_{2}, A_{2}\right) \text { and inf } z^{*}\left(A_{1}\right)<\inf z^{*}\left(A_{2}\right) .
\end{array}\right.
$$

It is easy to verify that $\preceq_{F, z^{*}}$ is reflexive, transitive, and antisymmetric.

The restriction of $\preceq_{F, z^{*}}$ to $X \times Y$ is denoted by $\preceq_{F, z^{*}}^{1}$ and is given by

$$
\left(x_{1}, y_{1}\right) \preceq_{F, z^{*}}^{1}\left(x_{2}, y_{2}\right): \Longleftrightarrow\left\{\begin{array}{l}
\left(x_{1}, y_{1}\right)=\left(x_{2}, y_{2}\right) \text { or } \\
\left(x_{1}, y_{1}\right) \preceq_{F}^{1}\left(x_{2}, y_{2}\right) \text { and } z^{*}\left(y_{1}\right)<z^{*}\left(y_{2}\right) .
\end{array}\right.
$$

The partial order $\preceq_{F, z^{*}}^{1}$ was introduced and used in [6, p. 913] (see also [5, p. 202]) for $F\left(x, x^{\prime}\right):=\left\{d\left(x, x^{\prime}\right) k^{0}\right\}$ with $k^{0} \in K \backslash(-\operatorname{cl} K)$, while $\preceq_{F, z^{*}}$ was introduced and used in [29]. A generalization can be found in [9, Section 7.2, p.131] where $z^{*}$ was replaced by a general function $f: Y \rightarrow \mathbb{R} \cup\{+\infty\}$ which is monotone wrt the second component of an order relation on $X \times Y$. Such order relations are basically two-component extensions of relations like the one defined in (25) below. Of course, for $z^{*}=0$ the relation $\preceq_{F, z^{*}}$ reduces to the equality relation on $X \times 2^{Y}$. If $z^{*} \in K^{\#}$ and $0 \notin F\left(x, x^{\prime}\right)$ for $x \neq x^{\prime}$ one has that $\preceq_{F, z^{*}}^{1}$ and $\preceq_{F}^{1}$ coincide.

We denote by $\preceq_{H, z^{*}}$ and $\preceq_{H, z^{*}}^{1}$ the partial orders $\preceq_{F_{H}, z^{*}}$ and $\preceq_{F_{H}, z^{*}}^{1}$, respectively.

\section{The Brezis-Browder principle}

A basic preliminary result in getting EVP type results revealed to be the Brezis-Browder principle. Consider $W$ a nonempty set and $t \subset W \times W$ a transitive relation; the fact that $\left(w, w^{\prime}\right)$ belongs to $t$ will be denoted by $w \preccurlyeq w^{\prime}$ or $w^{\prime} \succcurlyeq w$. Moreover, we shall identify $t$ and $\preceq$. As usual, we say that the sequence $\left(w_{n}\right)_{n \geq 1} \subset W$ is $\preccurlyeq$-decreasing if $w_{n+1} \preccurlyeq w_{n}$ for $n \geq 1 ;\left(w_{n}\right)_{n \geq 1} \subset W$ is strictly $\preccurlyeq$-decreasing if it is $\preccurlyeq$-decreasing and $w_{n+1} \neq w_{n}$ for $n \geq 1$. Moreover, we say that the nonempty set $A \subset W$ is bounded from below (or lower bounded, or minorized) (wrt $\preccurlyeq$ ) if there exists $v \in W$ such that $v \preccurlyeq w$ for all $w \in A$. We set $S_{\preccurlyeq}(w):=\left\{w^{\prime} \in W \mid w^{\prime} \preccurlyeq w\right\}$ for each $w \in W$; when there is no danger of confusion we write simply $S(w)$ instead of $S_{\preccurlyeq}(w)$. Of course, $S_{\preccurlyeq}: W \rightrightarrows W$ is a multifunction whose domain is $\operatorname{dom} S_{\preccurlyeq}:=\left\{w \in W \mid S_{\preccurlyeq}(w) \neq \emptyset\right\}$. Clearly $w^{\prime} \in S_{\preccurlyeq}(w) \Rightarrow S_{\preccurlyeq}\left(w^{\prime}\right) \subset S_{\preccurlyeq}(w)$, and $\operatorname{dom} S=W$ when $\preceq$ is a preoder.

The next assumption will be used often in the sequel.

(Ab) Any $\preccurlyeq$-decreasing sequence $\left(w_{n}\right)_{n \geq 1} \subset W$ is minorized.

Observe that condition $(\mathrm{Ab})$ is equivalent with the (apparently weaker) condition

(Ab') Any strictly $\preccurlyeq$-decreasing sequence $\left(w_{n}\right)_{n \geq 1} \subset W$ is minorized. 
Indeed, clearly, $\left.(\mathrm{Ab}) \Rightarrow(\mathrm{Ab})^{\prime}\right)$. Conversely, assume that $\left(\mathrm{Ab}\right.$ ') holds and take $\left(w_{n}\right)_{n \geq 1} \subset W$ $\mathrm{a} \preccurlyeq$-decreasing sequence. Set $P:=\left\{n \geq 1 \mid w_{n+1} \neq w_{n}\right\}$. If $P$ is finite, then there exists $\bar{n} \geq 1$ such that $w_{n+1}=w_{n}$ for $n \geq \bar{n}$, and so $w_{n}=w_{\bar{n}}$ for $n \geq \bar{n}$; in this case $w:=w_{\bar{n}} \leq w_{n}$ for all $n \geq 1$. Assume that $P$ is infinite. Then $P=\left\{n_{k} \mid k \geq 1\right\}$ where $n_{k+1}>n_{k}$ for $k \in \mathbb{N} \backslash\{0\}$. Setting $w_{k}^{\prime}:=w_{n_{k}}$, we have that $w_{k+1}^{\prime} \in S\left(w_{k}^{\prime}\right) \backslash\left\{w_{k}^{\prime}\right)$ for $k \geq 1$. By (Ab') there exists $w \in W$ such that $w \preccurlyeq w_{k}^{\prime}$ for $k \geq 1$. But for $n \geq 1$ there exists $k \geq 1$ such that $n_{k} \geq n$, and so $w \preccurlyeq w_{k}^{\prime}=w_{n_{k}} \preccurlyeq w_{n}$.

Assume that $\preceq$ is a transitive relation on $W$ and $\phi: W \rightarrow \overline{\mathbb{R}}$. We say that $\phi$ is $\preceq$-increasing if $w_{1} \preccurlyeq w_{2}$ implies $\phi\left(w_{1}\right) \leq \phi\left(w_{2}\right) ; \phi$ is strictly $\preceq$-increasing if $w_{1} \preccurlyeq w_{2}$ and $w_{1} \neq w_{2}$ imply $\phi\left(w_{1}\right) \leq \phi\left(w_{2}\right)$.

Partial orders of the type $\preccurlyeq_{\phi}$ introduced in (25) below will be used several times in the sequel.

Lemma 3.1 Let $\preceq$ be a transitive relation on $W$, and let $\phi: W \rightarrow \overline{\mathbb{R}}$ be $\preceq$-increasing. Let us set

$$
w_{1} \preccurlyeq \phi w_{2}: \Longleftrightarrow\left[w_{1}=w_{2} \text {, or }\left[w_{1} \preccurlyeq w_{2} \text { and } \phi\left(w_{1}\right)<\phi\left(w_{2}\right)\right]\right] .
$$

Then the following assertions hold:

(i) $\preccurlyeq_{\phi}$ is a partial order on $W$ and $\phi$ is strictly $\preceq_{\phi}$-increasing; moreover, $\preccurlyeq_{\phi}=\preceq \cup \Delta_{W}$ whenever $\phi$ is strictly $\preceq$-increasing.

(ii) If $\bar{w} \in W$ is a minimal point of $W$ wrt $\preceq_{\phi}$, then $W \ni w^{\prime} \preccurlyeq \bar{w}$ implies $\phi\left(w^{\prime}\right)=\phi(\bar{w})$.

(iii) If $(W, \preceq)$ verifies condition $(\mathrm{Ab})$, then $\left(W, \preccurlyeq_{\phi}\right)$ verifies condition $(\mathrm{Ab})$, too.

Proof. (i) From its very definition $\preccurlyeq \phi$ is reflexive; moreover, using the transitivity of $\preccurlyeq$ and the fact that $\phi$ is $\preccurlyeq$-increasing, one gets immediately that $\preccurlyeq_{\phi}$ is transitive. Now, take $w_{1}$, $w_{2} \in W$ such that $w_{1} \preccurlyeq_{\phi} w_{2}$ and $w_{2} \preccurlyeq_{\phi} w_{1}$. Assuming that $w_{1} \neq w_{2}$, from (25) we get the contradiction $\phi\left(w_{1}\right)<\phi\left(w_{2}\right)<\phi\left(w_{1}\right)$. Hence $\preccurlyeq \phi$ is also antisymmetric, and so $\preccurlyeq \phi$ is a partial order. The fact that $\phi$ is strictly $\preceq_{\phi}$-increasing follows from the very definition of $\preceq_{\phi}$. The equality $\preccurlyeq_{\phi}=\preceq \cup \Delta_{W}$ is obvious when $\phi$ is strictly $\preceq$-increasing.

(ii) It is clear that $\phi:(W, \preccurlyeq \phi) \rightarrow \overline{\mathbb{R}}$ is strictly $\preccurlyeq_{\phi}$-increasing. Consider a minimal point $\bar{w} \in W$ wrt $\preceq_{\phi}$, and take $w^{\prime} \preccurlyeq \bar{w}$; then $\phi\left(w^{\prime}\right) \preccurlyeq \phi(\bar{w})$. If $\phi\left(w^{\prime}\right)<\phi(\bar{w})$ then $w^{\prime} \preccurlyeq \phi \bar{w}$ and $w^{\prime} \neq \bar{w}$, contradicting the minimality of $\bar{w}$ wrt $\preccurlyeq \phi$. Hence $\phi\left(w^{\prime}\right)=\phi(\bar{w})$.

(iii) Take $\left(w_{n}\right)_{n \geq 1} \subset W$ a $\preccurlyeq \phi$-decreasing sequence. Then $\left(w_{n}\right)_{n \geq 1}$ is $\preccurlyeq$-decreasing, and so there exists $\widetilde{w} \in E$ such that $\widetilde{w} \preccurlyeq w_{n}$ for $n \geq 1$; hence $\phi(\widetilde{w}) \leq \phi\left(w_{n}\right)$ for $n \geq 1$. If $\phi(\widetilde{w})=\phi\left(w_{n_{0}}\right)$ for some $n_{0} \geq 1$ then $w_{n}=w_{n_{0}}$ for $n \geq n_{0}$, and so $w:=w_{n_{0}} \preccurlyeq \phi w_{n}$ for $n \geq 1$. In the contrary case, $\phi(\widetilde{w})<\phi\left(w_{n}\right)$, and so $\widetilde{w} \preccurlyeq_{\phi} w_{n}$, for $n \geq 1$.

The next result is a slight extension of the celebrated Brezis-Browder principle.

Theorem 3.2 (BB-principle) Let $\preceq$ be a transitive relation on $W$ satisfying $(\mathrm{Ab})$, and let $\phi: W \rightarrow \mathbb{R}$ be a lower bounded $\preceq$-increasing function. Then

(i) for every $w \in \operatorname{dom} S_{\preceq}$ there exists $\bar{w} \in S_{\preceq}(w)$ such that $\phi\left(w^{\prime}\right)=\phi(\bar{w})$ for all $w^{\prime} \in$ $S_{\preceq}(\bar{w})$;

(ii) moreover, if $\phi$ is strictly $\preceq$-increasing, then $\preccurlyeq$ is antisymmetric and for every $w \in$ $\operatorname{dom} S_{\preceq}$ there exists $\bar{w} \in S_{\preceq}(w)$ such that $S_{\preceq}(\bar{w}) \subset\{\bar{w}\}$.

Proof. Using Lemma 3.1 (i) and (iii), we have that $\preceq_{\phi}$ is a partial order verifying (Ab) and $\phi$ is strictly $\preceq_{\phi}$-increasing. Let $w \in \operatorname{dom} S_{\preceq}$; using the second part of [1, Cor. 1] (for the reversed order), there exists a minimal point $\bar{w}$ of $W$ wrt $\preceq_{\phi}$ such that $\bar{w} \preceq_{\phi} w$. By Lemma 
3.1 (ii), $W \ni w^{\prime} \preccurlyeq \bar{w}$ implies $\phi\left(w^{\prime}\right)=\phi(\bar{w})$; hence (i) holds. Assume moreover, that $\phi$ is strictly $\preceq$-increasing and take $w^{\prime} \in S_{\preceq}(\bar{w}) \backslash\{\bar{w}\}$ (if possible). Then $w^{\prime} \preceq_{\phi} \bar{w}$, and so we get the contradiction $w^{\prime}=\bar{w}$ by the $\preceq_{\phi^{-}}$-minimality of $\bar{w}$. Hence (ii) holds, too.

As observed by Corneliu Ursescu (see [2, p. 120]) and Turinici (see [31]), the BB-principle is valid also for $\preceq$-increasing functions $\phi:(W, \preceq) \rightarrow \overline{\mathbb{R}}$. The corresponding version of Theorem 3.2 is the following result. For getting it (as in [2] and [31]) it is sufficient to consider the strictly increasing function $\varphi: \overline{\mathbb{R}} \rightarrow \mathbb{R}$ defined by $\varphi(t):=t /(1+|t|)$ for $t \in \mathbb{R}, \varphi( \pm \infty):= \pm 1$ and to apply Theorem 3.2 for $\varphi \circ \phi: W \rightarrow \mathbb{R}$.

Theorem 3.3 (extended BB-principle) Let $\preceq$ be a transitive relation on $W$ satisfying (Ab), and let $\phi: W \rightarrow \overline{\mathbb{R}}$ be $a \preceq$-increasing function. Then the conclusions of Theorem [3.2 hold.

Having in view several recent extensions of the BB-principle, we provide below a proof of Theorem 3.2 (i) without using Lemma 3.1] or [1, Cor. 1].

Alternative (direct) proof of Theorem 3.2 (i) [assertion (ii) follows immediately from (i)].

For $w \in W$ set $\phi_{w}:=\inf \{\phi(u) \mid u \in S(w)\}$ with the convention $\inf \emptyset:=+\infty$. Clearly, $-\infty<\bar{\gamma}:=\inf \phi(W) \leq \phi_{w} \leq \phi(w)$ for $w \in \operatorname{dom} S$, and $\phi_{w}=\infty$ for $w \notin \operatorname{dom} S$; moreover, for $w^{\prime} \preccurlyeq w$ we have that $\phi_{w^{\prime}} \geq \phi_{w}$. Consider $\left.\left(\varepsilon_{n}\right)_{n \geq 1} \subset\right] 0, \infty\left[\right.$ with $\varepsilon_{n} \rightarrow 0$. Fix $w \in \operatorname{dom} S$ and set $w_{0}:=w$. If $\phi_{w_{0}}=\phi\left(w_{0}\right), \bar{w}:=w_{0}$ is the desired element. In the contrary case, there exists $w_{1} \in S\left(w_{0}\right)$ such that $\phi\left(w_{1}\right)<\min \left\{\phi\left(w_{0}\right), \phi_{w_{0}}+\varepsilon_{1}\right\}$. If $w_{1} \notin \operatorname{dom} S$ or $\phi_{w_{1}}=\phi\left(w_{1}\right)$, $\bar{w}:=w_{1}$ is the desired element. Continuing in this way, either the process stops at the step $n \geq 1$ because $w_{n} \notin \operatorname{dom} S$ or $\phi_{w_{n}}=\phi\left(w_{n}\right)$, in which case $\bar{w}:=w_{n}$ is the desired element, or, else we get the (strictly) $\preccurlyeq$-decreasing sequence $\left(w_{n}\right)_{n \geq 1}$ such that

$$
\phi\left(w_{n+1}\right)<\min \left\{\phi\left(w_{n}\right), \phi_{w_{n}}+\varepsilon_{n+1}\right\} \quad \forall n \geq 0 .
$$

Therefore, $\left(w_{n}\right)_{n \geq 0}$ is $\preccurlyeq$-decreasing, $\left(\phi_{w_{n}}\right)_{n \geq 0}$ is increasing and $\left(\phi\left(w_{n}\right)\right)_{n \geq 0}$ is strictly decreasing (in $\mathbb{R}$ ) with

$$
\phi_{w_{n}} \leq \phi_{w_{n+1}} \leq \phi\left(w_{n+1}\right)<\phi_{w_{n}}+\varepsilon_{n+1} \quad \forall n \geq 0
$$

Hence

$$
\phi\left(w_{n+1}\right)<\phi\left(w_{n}\right) \text { and } 0 \leq \phi\left(w_{n+1}\right)-\inf \left\{\phi(u) \mid u \in S\left(w_{n}\right)\right\}<\varepsilon_{n+1} \quad \forall n \geq 0 .
$$

It follows that there exists $\gamma \in \mathbb{R}$ such that $\lim _{n \rightarrow \infty} \phi_{w_{n}}=\lim _{n \rightarrow \infty} \phi\left(w_{n}\right)=\gamma$, and so $\phi_{w_{n}} \leq \gamma \leq \phi\left(w_{n}\right)$ for $n \geq 0$. By (Ab') we get $\bar{w} \in W$ such that $\bar{w} \preccurlyeq w_{n}$, and so $S(\bar{w}) \cup\{\bar{w}\} \subset$ $S\left(w_{n}\right) \subset S\left(w_{0}\right)$ for every $n \geq 0$; in particular, $\bar{w} \in S\left(w_{0}\right)$. Hence for $w^{\prime} \in S(\bar{w}) \cup\{\bar{w}\}$ we have that $\phi_{w_{n}} \leq \phi\left(w^{\prime}\right) \leq \phi\left(w_{n}\right)$ for all $n \geq 1$. Taking the limit for $n \rightarrow \infty$ we get $\phi\left(w^{\prime}\right)=\phi(\bar{w})$ $(=\gamma)$ for $w^{\prime} \in S(\bar{w})$. Hence $\bar{w}$ is the desired element.

The above proof of Theorem 3.2 shows that the next result holds true.

Theorem 3.4 Let $\preceq$ be a transitive relation on $W$ and let $\phi: W \rightarrow \mathbb{R}$ be a lower bounded $\preceq$-increasing function. Assume that

(AQ) any strictly $\preccurlyeq$-decreasing sequence $\left(w_{n}\right)_{n \geq 1} \subset W$ with $\left(\phi\left(w_{n}\right)\right)_{n \geq 1}$ strictly decreasing and $\phi\left(w_{n+1}\right)-\inf \left\{\phi(v) \mid v \preccurlyeq w_{n}\right\} \rightarrow 0$ is minorized.

Then assertion (i) of Theorem 3.2 holds. Moreover, if $\phi$ is strictly $\preceq$-increasing, then assertion (ii) of Theorem 3.2 holds, too. 
Remark 3.1 (a) Theorem 5.1 in 2 follows from the first part of Theorem 3.4 applying it for the reversed order and $\phi:=-S$, because in [2] it is assumed that $\preccurlyeq$ is a partial order and any increasing sequence $\left(x_{n}\right)_{n \geq 1}$ with $\left(S\left(x_{n}\right)\right)_{n \geq 1}$ strictly increasing is bounded above.

(b) Theorems 2.1 in [25], [26] and [27] follow applying Theorem 3.4 for $W:=S\left(x_{1}\right)$ with $x_{1} \in S\left(x_{0}\right) \backslash\left\{x_{0}\right\}$ such that $\eta\left(x_{1}\right)<\infty$ and $\phi:=\left.\eta\right|_{W}$ because it is assumed that every strictly $\preccurlyeq$-decreasing sequence $\left(w_{n}\right)_{n \geq 1} \subset W$ with $\phi\left(w_{n+1}\right)-\inf \left\{\phi(v) \mid v \preccurlyeq w_{n}\right\} \rightarrow 0$ is minorized. More precisely, [25, Th. 2.1] and [27, Th. 2.1] follow directly using the second part of Theorem 3.4 because $\phi=\left.\eta\right|_{W}$ is strictly increasing. In the case of [26, Th. 2.1], using the first part of Theorem 3.4, one obtains some $\bar{w} \in W$ such that $\phi\left(w^{\prime}\right)=\phi(\bar{w})$ for $w^{\prime} \in S(\bar{w})$. Because, by condition (B), for $z_{1}, z_{2} \in S(x)$ with $x \in S\left(x_{0}\right)$ we have that $\min \left\{\eta\left(z_{1}\right), \eta\left(z_{2}\right)\right\}<\eta(x), S(\bar{w})$ contains at most one element $\widetilde{w}$. If $S(\bar{w}) \subset\{\bar{w}\}$ we take $\widehat{w}:=\bar{w}$. In the contrary case $\widetilde{w} \neq \bar{w}$ and $S(\bar{w})=\{\widetilde{w}\}$, whence $S(\widetilde{w}) \subset S(\bar{w})=\{\widetilde{w}\}$. Taking $\widehat{w}:=\widetilde{w}$, the conclusion of [26, Th. 2.1] holds.

As O. Cârjă mentions in [2, p. 120], the use of strict monotonicity of the sequence $\left(S\left(x_{n}\right)\right)$ in the condition corresponding to $(\mathrm{Ab})$ of the usual version of the BB-principle is due to Corneliu Ursescu.

In the sequel $\preceq$ is a preorder if not stated explicitly otherwise.

Having the metric space $(X, d)$, the nonempty set $Z$, and $\mathcal{A}$ a nonempty subset of $X \times Z$ preordered by $\preceq$, consider the conditions:

(C0) $\forall\left(\left(x_{n}, z_{n}\right)\right)_{n \geq 1} \subset \mathcal{A} \preceq$-decreasing : $\left(x_{n}\right)_{n \geq 1}$ is Cauchy and $\exists(x, z) \in \mathcal{A}$ such that $(x, z) \preceq\left(x_{n}, z_{n}\right) \forall n \geq 1$.

(C'0) $\forall\left(\left(x_{n}, z_{n}\right)\right)_{n \geq 1} \subset \mathcal{A} \preceq$-decreasing : $\exists(x, z) \in \mathcal{A}$ such that $x_{n} \rightarrow x$ and $(x, z) \preceq\left(x_{n}, z_{n}\right)$ $\forall n \geq 1$.

(Ca) $\forall\left(\left(x_{n}, z_{n}\right)\right)_{n \geq 1} \subset \mathcal{A} \preceq$-decreasing : $\left(x_{n}\right)_{n \geq 1}$ is Cauchy.

(Cb) $\forall\left(\left(x_{n}, z_{n}\right)\right)_{n \geq 1} \subset \mathcal{A} \preceq$-decreasing : $\exists(x, z) \in \mathcal{A}$ such that $(x, z) \preceq\left(x_{n}, z_{n}\right) \forall n \geq 1$.

(C1) $\forall\left(\left(x_{n}, z_{n}\right)\right)_{n \geq 1} \subset \mathcal{A} \preceq$-decreasing with $\left(x_{n}\right)_{n \geq 1}$ Cauchy : $\exists(x, z) \in \mathcal{A}$ such that $(x, z) \preceq$ $\left(x_{n}, z_{n}\right) \forall n \geq 1$.

(C'1) $\forall\left(\left(x_{n}, z_{n}\right)\right)_{n \geq 1} \subset \mathcal{A} \preceq$-decreasing with $x_{n} \rightarrow x \in X: \exists z \in Z$ such that $(x, z) \in \mathcal{A}$ and $(x, z) \preceq\left(x_{n}, z_{n}\right) \forall n \geq 1$.

(Ca1) $\forall\left(\left(x_{n}, z_{n}\right)\right)_{n \geq 1} \subset \mathcal{A} \preceq$-decreasing with $\left(x_{n}\right)_{n \geq 1}$ Cauchy : $\left(x_{n}\right)$ is convergent.

Clearly, condition $(\mathrm{Cb})$ is nothing else than condition $(\mathrm{Ab})$ written for $(\mathcal{A}, \preceq)$. Furthermore, $(\mathrm{Ca} 1)$ is automatically verified whenever $(X, d)$ is complete.

When $X$ is a singleton $\left\{x_{0}\right\}$ the conditions above, excepting (Ca) and (Ca1) which are automatically satisfied, reduce to $(\mathrm{Ab})$ for $(\mathcal{A}, \preceq)$, or, equivalently, for $(W, \preccurlyeq)$, where $W=$ $\operatorname{Pr}_{Z}(\mathcal{A}):=\{z \in Z \mid \exists x \in X:(x, z) \in A\}$ and $w \preccurlyeq w^{\prime}$ if $\left(x_{0}, w\right) \preceq\left(x_{0}, w^{\prime}\right)$ for $w, w^{\prime} \in W$.

When $Z$ is a singleton $\left\{z_{0}\right\}$, the preorder $\preceq$ on $\mathcal{A} \subset X \times Z$ is uniquely determined by the preorder $\preccurlyeq$ on $\operatorname{Pr}_{X}(\mathcal{A})$ defined by $x \preccurlyeq x^{\prime}$ if $\left(x, z_{0}\right) \preceq\left(x^{\prime}, z_{0}\right)$. So, when $Z$ is a singleton, the conditions above reduce to the following ones on the preordered metric space $(X, d, \preccurlyeq)$ (replacing $X$ by $\operatorname{Pr}_{X}(\mathcal{A})$ if necessary): 
(A0) $\forall\left(x_{n}\right)_{n \geq 1} \subset X \preccurlyeq$-decreasing : $\left(x_{n}\right)_{n \geq 1}$ is Cauchy and $\exists x \in X$ such that $x \preccurlyeq x_{n} \forall n \geq 1$.

(A'0) $\forall\left(x_{n}\right)_{n \geq 1} \subset X \preccurlyeq$-decreasing : $\exists x \in X$ such that $x_{n} \rightarrow x$ and $x \preccurlyeq x_{n} \forall n \geq 1$.

(Aa) $\forall\left(x_{n}\right)_{n \geq 1} \subset X \preccurlyeq$-decreasing : $\left(x_{n}\right)_{n \geq 1}$ is Cauchy.

(Ab) $\forall\left(x_{n}\right)_{n \geq 1} \subset X \preccurlyeq$-decreasing : $\exists x \in X$ such that $x \preccurlyeq x_{n} \forall n \geq 1$.

(A1) $\forall\left(x_{n}\right)_{n \geq 1} \subset X \preccurlyeq$-decreasing with $\left(x_{n}\right)_{n \geq 1}$ Cauchy : $\exists x \in X$ such that $x \preccurlyeq x_{n} \forall n \geq 1$.

(A'1) $\forall\left(x_{n}\right)_{n \geq 1} \subset X \preccurlyeq$-decreasing with $x_{n} \rightarrow x \in X: x \preccurlyeq x_{n} \forall n \geq 1$.

(Aa1) $\forall\left(x_{n}\right)_{n \geq 1} \subset X \preccurlyeq$-decreasing with $\left(x_{n}\right)_{n \geq 1}$ Cauchy : $\left(x_{n}\right)$ is convergent.

Taking into account Remark 3.3 below, condition (Aa) means that $\preccurlyeq$ is regular in the sense of [9, p. 68], while (A'1) means that all lower sections of $X$ wrt $\preccurlyeq$ are $\preccurlyeq$-lower closed. When $(X, d, \preccurlyeq)$ verifies (Aa1), in [9, p. 67] it is said that $(X, d)$ is $\preccurlyeq$-complete. Condition (Aa1) is also related to dynamical completeness of $(X, d)$ in the sense of [25, Def. 3.1].

Proposition 3.5 Among the conditions above we have the following relations:

$$
\begin{gathered}
(\mathrm{C} 0) \Longleftrightarrow(\mathrm{Ca}) \wedge(\mathrm{Cb}) \Longleftrightarrow(\mathrm{Ca}) \wedge(\mathrm{C} 1), \\
(\mathrm{Cb}) \Longrightarrow(\mathrm{C} 1), \quad\left(\mathrm{C}^{\prime} 0\right) \Longrightarrow\left(\mathrm{C}^{\prime} 1\right), \\
\left(\mathrm{C}^{\prime} 0\right) \Longrightarrow(\mathrm{C} 0), \quad\left(\mathrm{C}^{\prime} 1\right) \wedge(\mathrm{Ca} 1) \Longrightarrow(\mathrm{C} 1) .
\end{gathered}
$$

In general, $\left(\mathrm{C}^{\prime} 1\right) \nRightarrow(\mathrm{C} 1)$, and the converse implications in (27) and (28) are not true, even if $(X, d)$ is complete and $Z$ is a singleton.

Proof. The implications in (26), (27) and (28) are almost obvious. The fact that the implication $\left(\mathrm{C}^{\prime} 1\right) \Rightarrow(\mathrm{C} 1)$ in general is not true when $(X, d)$ is not complete is shown in Example 3.6 (a). The fact that the converse implications in (27) and (28) are not true in general follows from Example 3.6 (b) and (c), respectively.

Example 3.6 (a) Consider $X:=] 0,1]$ endowed with the metric $d$ defined by $d\left(x, x^{\prime}\right):=$ $\left|x-x^{\prime}\right|$ and the order $\preccurlyeq$ defined by $x \preccurlyeq x^{\prime}$ if $x^{\prime}-x \in \mathbb{R}_{+}$. Take $\left(x_{n}\right)_{\geq 1} \subset X$ a $\preccurlyeq$-decreasing sequence with $x_{n} \rightarrow x \in X$. On one hand, since the metric and order on $X$ are induced by the usual metric and order of $\mathbb{R}$, we have that $x \leq x_{n}$ for $n \geq 1$, and so (A'1) holds. On the other hand, the sequence $(1 / n)_{n \geq 1} \subset X$ is Cauchy and $\preccurlyeq$-decreasing, but assuming that there exists $x \in X$ such that $x \preccurlyeq 1 / n$ (thus $x \leq 1 / n$ ) for $n \geq 1$, we get the contradiction $x \leq 0$. Hence (A1) does not hold.

(b) Consider $X:=[0,1] \subset \mathbb{R}, d: X \times X \rightarrow \mathbb{R}$ defined by $d\left(x, x^{\prime}\right):=0$ for $x=x^{\prime}$, $d\left(x, x^{\prime}\right):=1$ for $x \neq x^{\prime}$, and $x \preccurlyeq x^{\prime}$ if $x^{\prime}-x \in \mathbb{R}_{+}$for $x, x^{\prime} \in X$; clearly, $(X, d)$ is a complete metric space. It is obvious that (A1) and (A'1) hold because $\left(x_{n}\right)_{n \geq 1} \subset X$ is Cauchy (convergent) iff $\left(x_{n}\right)_{n \geq 1}$ is constant for large $n$, but neither (AO), nor (A'O), holds; just take the sequence $\left(x_{n}\right)_{n \geq 1}:=(1 / n)_{n \geq 1} \subset X$.

(c) Consider $X:=\{-1\} \cup[0,1] \subset \mathbb{R}, d: X \times X \rightarrow \mathbb{R}$ defined by $d\left(x, x^{\prime}\right):=\left|x-x^{\prime}\right|$, and $x \preccurlyeq x^{\prime}$ if either $x^{\prime}=0$, or $x, x^{\prime} \in X \backslash\{0\}$ and $x^{\prime}-x \in \mathbb{R}_{+}$for $x, x^{\prime} \in X$; clearly, $(X, d)$ is a complete metric space. Because $-1 \preccurlyeq x$ for every $x \in X$, (A1) clearly holds. Also (A0) holds. Indeed, take $\left(x_{n}\right)_{n \geq 1} \subset X$ a -decreasing sequence. As observed above, $-1 \preccurlyeq x_{n}$ for every $n \geq 1$. If $x_{n_{0}} \neq 0$ for some $n_{0} \geq 1$, then $\left(x_{n}\right)_{n \geq n_{0}} \subset X \backslash\{0\}$, and so $\left(x_{n}\right)_{n \geq n_{0}}$ as a 
sequence in $\mathbb{R}$ with its usual order is decreasing and bounded from below by -1 , and so it is convergent to an element in $X$ (hence $\left(x_{n}\right)_{n \geq 1}$ is Cauchy in $(X, d)$ ). In the contrary case, $x_{n}=0$ for $n \geq 1$, and so $\left(x_{n}\right)_{n \geq 1}$ is Cauchy. Moreover, (A'1) and (A'0) do not hold because $(1 / n)_{n \geq 1} \subset X$ is $\preccurlyeq$-decreasing and converges to 0 , but $0 \nprec 1 / n$ for every $n \geq 1$.

Using Theorem 3.3 we get the next result; it is close to [9, Th. 21], and is the prototype of the results in this paper, some of them being direct consequences of it.

Theorem 3.7 Let $(X, d)$ be a metric space, $Z$ a nonempty set, and let $\mathcal{A} \subset X \times Z$ be a nonempty set preordered by $\preceq$ which verifies $(\mathrm{C} 0)$. Then for every $(x, z) \in \mathcal{A}$ there exists $(\bar{x}, \bar{z}) \in \mathcal{A}$ such that $(\bar{x}, \bar{z}) \preceq(x, z)$ and $\mathcal{A} \ni\left(x^{\prime}, z^{\prime}\right) \preceq(\bar{x}, \bar{z}) \Rightarrow x^{\prime}=\bar{x}$.

Proof. For $(x, z) \in \mathcal{A}$ set $S(x, z):=\left\{\left(x^{\prime}, z^{\prime}\right) \in \mathcal{A} \mid\left(x^{\prime}, z^{\prime}\right) \preceq(x, z)\right\}$; moreover, consider

$$
\phi: \mathcal{A} \rightarrow \overline{\mathbb{R}}, \quad \phi(x, z):=\operatorname{diam} P_{X}(S(x, z)) .
$$

Since $S\left(x^{\prime}, z^{\prime}\right) \subset S(x, z)$ when $(x, z),\left(x^{\prime}, z^{\prime}\right) \in \mathcal{A}$ with $\left(x^{\prime}, z^{\prime}\right) \preceq(x, z), \phi$ is $\preceq$-increasing. Because of condition (Ab), the hypotheses of Theorem 3.3 are verified.

Take $(x, z) \in \mathcal{A}$. Using Theorem 3.3 we get $(\bar{x}, \bar{z}) \in \mathcal{A}$ such that $(\bar{x}, \bar{z}) \preceq(x, z)$ and $\mathcal{A} \ni\left(x^{\prime}, z^{\prime}\right) \preceq(\bar{x}, \bar{z}) \Rightarrow \phi\left(x^{\prime}, z^{\prime}\right)=\phi(\bar{x}, \bar{z})=: \alpha \in[0, \infty]$. We claim that $\alpha=0$, and so the conclusion holds.

Assume that $\alpha>0$ and take $0<\beta<\frac{1}{2} \alpha$. Then there exists $\left(x_{1}, z_{1}\right) \in S(\bar{x}, \bar{z})$ such that $d\left(x_{1}, \bar{x}\right)>\beta$; else, $d\left(x^{\prime}, \bar{x}\right) \leq \beta$ for $\left(x^{\prime}, z^{\prime}\right) \in S(\bar{x}, \bar{z})$, and so $\phi(\bar{x}, \bar{z})=\operatorname{diam} \operatorname{Pr}_{X}(S(\bar{x}, \bar{z})) \leq$ $2 \beta<\alpha$, a contradiction. Since $\left(x_{1}, z_{1}\right) \preceq(\bar{x}, \bar{z}), \operatorname{diam} \operatorname{Pr}_{X}\left(S\left(x_{1}, z_{1}\right)\right)=\phi\left(x_{1}, z_{1}\right)=\alpha$. As before, we find $\left(x_{2}, z_{2}\right) \preceq\left(x_{1}, z_{1}\right)(\preceq(\bar{x}, \bar{z}))$ with $d\left(x_{2}, x_{1}\right)>\beta$. Continuing in this way we get the $\preceq$-decreasing sequence $\left(\left(x_{n}, z_{n}\right)\right)_{n \geq 1} \subset \mathcal{A}$ such that $d\left(x_{n+1}, x_{n}\right)>\beta$ for all $n \geq 1$. By $(\mathrm{C} 0),\left(x_{n}\right)_{n \geq 1}$ is Cauchy, and so we get the contradiction $\beta<d\left(x_{n+1}, x_{n}\right) \rightarrow 0$. Hence $\alpha=0$, and so $\left(x^{\prime}, z^{\prime}\right) \in S(\bar{x}, \bar{z})$ implies $x^{\prime}=\bar{x}$.

Alternative proof of Theorem 3.7 (without using BB-principle).

Fix $(x, z) \in \mathcal{A}$; with the notation above, we have to show that there exists $(\bar{x}, \bar{z}) \in S(x, z)$ with $P_{X}(S(\bar{x}, \bar{z}))=\{\bar{x}\}$.

Starting with $\left(x_{1}, z_{1}\right):=(x, z) \in \mathcal{A}$, define a sequence $\left(\left(x_{n}, z_{n}\right)\right)_{n \geq 1} \subseteq \mathcal{A}$ by picking $x_{n+1} \in P_{X}\left(S\left(x_{n}, z_{n}\right)\right)$ satisfying

$$
d\left(x_{n+1}, x_{n}\right) \geq \min \left(1, \sup \left\{d\left(x, x_{n}\right) \mid x \in P_{X}\left(S\left(x_{n}, z_{n}\right)\right)\right\}-1 / n\right),
$$

and $z_{n+1} \in Z$ such that $\left(x_{n+1}, z_{n+1}\right) \in S\left(x_{n}, z_{n}\right)$.

Since $\left(\left(x_{n}, z_{n}\right)\right)_{n \geq 1}$ is $\preceq$-decreasing, by $(\mathrm{C} 0)$, the sequence $\left(x_{n}\right)_{n \geq 1}$ is Cauchy and there exists $(\bar{x}, \bar{z}) \in \mathcal{A}$ such that $(\bar{x}, \bar{z}) \preceq\left(x_{n}, z_{n}\right)$ for $n \geq 1$; in particular $(\bar{x}, \bar{z}) \preceq\left(x_{1}, z_{1}\right)=(x, z)$. It follows that $d\left(x_{n}, x_{n+1}\right) \rightarrow 0$, and so $d\left(x_{n}, x_{n+1}\right)<1$ for large $n$. These together with (29) imply that diam $P_{X}\left(S\left(x_{n}, z_{n}\right)\right) \rightarrow 0$. Since $S(\bar{x}, \bar{z}) \subset S\left(x_{n}, z_{n}\right)$ for $n \geq 1$, we obtain that diam $P_{X}(S(\bar{x}, \bar{z}))=0$. Hence $P_{X}(S(\bar{x}, \bar{z}))=\{\bar{x}\}$ because $(\bar{x}, \bar{z}) \in S(\bar{x}, \bar{z})$. The proof is complete.

Lemma 3.8 Let $(X, d)$ be a metric space, $Z$ a nonempty set, and $\mathcal{A} \subset X \times Z$ a nonempty set preordered by $\preceq$. Then condition (Ca) holds if and only if for every $\preceq$-decreasing sequence $\left(\left(x_{n}, z_{n}\right)\right)_{n \geq 1} \subseteq \mathcal{A}$ the sequence $\left(x_{n}\right)$ is asymptotic, that is $d\left(x_{n}, x_{n+1}\right) \rightarrow 0$. 
Proof. Of course, if $\left(\left(x_{n}, z_{n}\right)\right)_{n \geq 1} \subseteq \mathcal{A}$ verifies condition (Ca) then $d\left(x_{n}, x_{n+1}\right) \rightarrow 0$. Conversely, consider the $\preceq$-decreasing sequence $\left(\left(x_{n}, z_{n}\right)\right)_{n>1} \subseteq \mathcal{A}$ with $d\left(x_{n}, x_{n+1}\right) \rightarrow 0$. Suppose that $\left(x_{n}\right)$ is not Cauchy. Then there exist $\delta>0$ and a strictly increasing sequence $\left(n_{l}\right)_{l \geq 1} \subseteq \mathbb{N}^{*}$ such that $d\left(x_{n_{l+1}}, x_{n_{l}}\right) \geq \delta$ for all $l \geq 1$. Since $\preceq$ is transitive, $\left(\left(x_{n_{l}}, z_{n_{l}}\right)\right)_{l \geq 1}$ is $\preceq$-decreasing, and so $\left(x_{n_{l}}\right)_{l \geq 1}$ is asymptotic, that is $d\left(x_{n_{l+1}}, x_{n_{l}}\right) \rightarrow 0$. This obvious contradiction ends the proof.

Note that one must add that $(X, d)$ is complete in [9, Th. 21].

Indeed, take $X:=Y:=\mathbb{R}_{+} \backslash\{0\}, X$ endowed with the usual metric and $X \times Y$ endowed with the order defined by $(x, y) \preceq\left(x^{\prime}, y^{\prime}\right): \Longleftrightarrow\left[x \leq x^{\prime}, y \leq y^{\prime}\right]$; take also $M:=\{(x, x) \mid x>$ $0\}$. Clearly the hypothesis of [9, Th. 21] is satisfied, but its conclusion does not hold.

Taking into account (28), the next result is an obvious consequence of Theorem 3.7, it is stated in [9, Th. 23] under the supplementary assumption that $(X, d)$ is complete.

Corollary 3.9 Let $(X, d)$ be a metric space, $Z$ a nonempty set, and let $\emptyset \neq \mathcal{A} \subset X \times Z$ be preordered by $\preceq$. Assume that $(\mathcal{A}, \preceq)$ verifies condition $\left(\mathrm{C}^{\prime} 0\right)$. Then the conclusion of Theorem 3.7 holds.

Another consequence of Theorem 3.7 is the following result.

Corollary 3.10 Let $(X, d)$ be a metric space, preordered by $\preccurlyeq$, for which (A0) holds. Then for every $x \in X$ there exists $\bar{x} \in X$ such that $\bar{x} \preccurlyeq x$ and $X \ni x^{\prime} \preccurlyeq \bar{x} \Rightarrow x^{\prime}=\bar{x}$.

Proof. Take $\mathcal{A}:=X \times\{0\}$ and set $(x, 0) \preceq\left(x^{\prime}, 0\right)$ if $x, x^{\prime} \in X$ and $x \preccurlyeq x^{\prime}$. Clearly $\preceq$ is a preorder on $\mathcal{A}$ and condition (C0) in Theorem 3.7 is verified. Applying Theorem 3.7 we get the conclusion.

From the preceding result we get immediately the next one formulated by Liu and $\mathrm{Ng}$ in [19, Lem. 2.2].

Corollary 3.11 Let $(X, d)$ be a metric space, preordered by $\preccurlyeq$, for which (A'0) holds. Then for every $x \in X$ there exists $\bar{x} \in X$ such that $\bar{x} \preccurlyeq x$ and $X \ni x^{\prime} \preccurlyeq \bar{x} \Rightarrow x^{\prime}=\bar{x}$.

Remark 3.2 Corollary 3.10 is slightly more general than Corollary 3.11, To see this take $X:=\{-1\} \cup\{1 / n \mid n \in \mathbb{N} \backslash\{0\}\}$ endowed with the metric $d$ defined by $d\left(x, x^{\prime}\right):=\left|x-x^{\prime}\right|$ and the order $\preccurlyeq$ defined by $x \preccurlyeq x^{\prime}$ if $x^{\prime}-x \in \mathbb{R}_{+}$. Clearly the sequence $(1 / n)_{n \geq 1} \subset X$ is $\preccurlyeq$-decreasing, but not convergent.

As observed in [19], Corollary 3.11 is a reformulation of the next result stated by Hamel and Tammer in [13, Th. 2.2]; this result was stated previously, in equivalent forms, by Turinici in [30, Th. 1] and by Hamel in [9, Th. 16].

Corollary 3.12 Let $(X, d)$ be a metric space, preordered by $\preccurlyeq$ such that $(X, d)$ is $\preccurlyeq$-complete, that is every $\preccurlyeq$-decreasing Cauchy sequence $\left(x_{n}\right)_{n \geq 1} \subseteq X$ is convergent. Assume that

(i) $S(x):=\left\{x^{\prime} \in X \mid x^{\prime} \preccurlyeq x\right\}$ is $\preccurlyeq$-lower closed for every $x \in X$, that is for every $\preccurlyeq$-decreasing sequence $\left(x_{n}\right)_{n \geq 1} \subseteq S(x)$ with $x_{n} \rightarrow u$ one has $u \in S(x)$, and

(ii) any $\preccurlyeq$-decreasing sequence $\left(x_{n}\right)_{n \geq 1} \subseteq X$ is asymptotic.

Then for every $x \in X$ there exists $\bar{x} \in X$ such that $\bar{x} \preccurlyeq x$ and $S(\bar{x})=\{\bar{x}\}$. 
Remark 3.3 Conditions (i) and (ii) in Corollary 3.12 are equivalent to (A'1) and (Aa), respectively.

Indeed, assume that (i) in Corollary 3.12 holds and take $\left(x_{n}\right)_{n \geq 1} \subseteq X$ a $\preccurlyeq$-decreasing sequence with $x_{n} \rightarrow x \in X$. Since $\left(x_{n}\right)_{n \geq p} \subseteq S\left(x_{p}\right)$ and $S\left(x_{p}\right)$ is $\preccurlyeq$-lower closed, we have that $x \in S\left(x_{p}\right)$, and so $x \preccurlyeq x_{p}$ for every $p$. Conversely, assume that (A'1) holds and take $\left(x_{n}\right)_{n \geq 1} \subseteq S(x)$ a $\preccurlyeq$-decreasing sequence with $x_{n} \rightarrow u$. Then $u \preccurlyeq x_{1} \preccurlyeq x$, whence $u \in S(x)$. The equivalence of (ii) and (Aa) follows immediately from Lemma 3.8.

The equivalence of (ii) and (Aa) from the previous remark is established in [9, Prop. 41].

Note that in Corollary 3.12 (and Corollary [3.11) $\preccurlyeq$ is in fact anti-symmetric (as observed in [9, Prop. 40] and [13, Prop. 2.1]). Indeed, take $x, x^{\prime} \in X$ with $x \preccurlyeq x^{\prime}$ and $x^{\prime} \preccurlyeq x$. Then the sequence $\left(x_{n}\right)_{n \geq 1}$ defined by $x_{2 n}:=x$ and $x_{2 n-1}:=x^{\prime}$ is $\preccurlyeq$-decreasing; by (ii) we get $d\left(x, x^{\prime}\right)=d\left(x_{n}, x_{n+1}\right) \rightarrow 0$, and so $x=x^{\prime}$.

Note also that Corollary 3.12 is slightly more general than the Dancs-Hegedüs-Medvegyev Theorem (see [3, Th. 3.1]), in which $(X, d)$ is assumed to be complete instead of being $\preccurlyeq-$ complete and $S(x)$ is assumed to be closed instead of being $\preccurlyeq$-closed.

The next result is very easy to prove, so we omit its proof.

Proposition 3.13 Let $(Z, \preceq)$ be a preordered set. For any $A_{1}, A_{2} \in 2^{Z}$ let us set

$$
A_{1} \preceq^{l} A_{2}: \Longleftrightarrow \quad\left[\forall z_{2} \in A_{2}, \exists z_{1} \in A_{1}: z_{1} \preceq z_{2}\right] .
$$

Then $\preceq^{l}$ is a preorder on $2^{Z}$. Moreover, for $z_{1}, z_{2} \in Z$, and $A, A_{1}, A_{2} \in 2^{Z}$ we have that

$$
\left\{z_{1}\right\} \preceq^{l}\left\{z_{2}\right\} \Leftrightarrow z_{1} \preceq z_{2}, \quad Z \preceq^{l} A \preceq^{l} \emptyset, \quad\left[A_{2} \neq \emptyset, A_{1} \preceq^{l} A_{2}\right] \Rightarrow A_{1} \neq \emptyset .
$$

In this generality, the preorder $\preceq^{l}$ was probably discussed for the first time by Hamel in [9, (2.6)], at least in the context of variational analysis/optimization. However, there are many precursors: the interested reader is referred to [10] for a more thorough survey and detailed references.

An important example of the above construction, already discussed in [17] on a topological vector space ordered by a convex cone with non-empty interior, is the following: Let $Y$ be a real vector space and $K \subset Y$ a convex cone. Recall that the preorder $\leq_{K}$ determined by $K$ on $Y$ is defined by $y_{1} \leq_{K} y_{2}: \Longleftrightarrow y_{2}-y_{1} \in K$. The preorder on $2^{Y}$ corresponding to $\leq_{K}$ using the definition in (30) is the one given by $A_{1} \leq_{K}^{l} A_{2}: \Longleftrightarrow A_{2} \subset A_{1}+K$ (see Section 2). Of course, $\leq_{K}$ is a partial order on $Y$ if and only if $K$ is pointed (that is $K \cap(-K)=\{0\}$ ), but $\leq_{K}^{l}$ is a partial order if and only if $K=\{0\}$. Indeed, $\{0\} \leq_{K}^{l} K \leq_{K}^{l}\{0\}$.

Another application of Theorem $[3.3$ is the following result involving the set relation $\preceq l$.

Corollary 3.14 Let $(Z, \preceq)$ be a preordered set, and $\phi:(Z, \preceq) \rightarrow \overline{\mathbb{R}} a \preceq$-increasing function. Consider the preorder $\preceq^{l}$ on $2^{Z}$ defined in (30). For $A_{1}, A_{2} \in 2^{Z}$ let us set

$$
A_{1} \preceq_{\phi}^{l} A_{2}: \Longleftrightarrow \quad\left[A_{1}=A_{2} \text { or }\left[A_{1} \preceq^{l} A_{2} \text { and } \inf \phi\left(A_{1}\right)<\inf \phi\left(A_{2}\right)\right]\right] .
$$

Then $\preceq_{\phi}^{l}$ is a partial order on $2^{Z}$. Furthermore, let $\mathcal{A} \subset 2^{Z}$ be a nonempty set such that $\left(\mathcal{A}, \preceq^{l}\right)$ verifies condition (Ab). Then for every $A \in \mathcal{A}$ there exists a minimal set $\bar{A} \in \mathcal{A}$ wrt $\preceq_{\phi}^{l}$ such that $\bar{A} \preceq_{\phi}^{l} A ;$ moreover, $\mathcal{A} \ni A^{\prime} \preceq^{l} \bar{A}$ implies $\inf \phi\left(A^{\prime}\right)=\inf \phi(\bar{A})$. 
Proof. Consider the mapping

$$
\phi^{\prime}: 2^{Z} \rightarrow \overline{\mathbb{R}}, \quad \phi^{\prime}(A):=\inf \phi(A),
$$

where, as usual, $\inf \emptyset:=+\infty$. We claim that $\phi^{\prime}$ is $\preceq^{l}$-increasing. Indeed, take $A_{1}, A_{2} \in 2^{Z}$ such that $A_{1} \preceq^{l} A_{2}$. From the definition of $\preceq^{l}$, for $z_{2} \in A_{2}$ there exists $z_{1} \in A$ with $z_{1} \preceq z_{2}$, and so $\phi^{\prime}\left(A_{1}\right) \leq \phi\left(z_{1}\right) \leq \phi\left(z_{2}\right)$. Since $z_{2} \in A_{2}$ is arbitrary, we get $\phi^{\prime}\left(A_{1}\right) \leq \Phi^{\prime}\left(A_{2}\right)$. Using Lemma 3.1 for $W:=2^{Z}, \preccurlyeq:=\preceq^{l}$ and $\phi$ replaced by $\phi^{\prime}$, we obtain that $\preceq_{\phi}^{l}$ is a partial order on $2^{Z}$; moreover, $\phi^{\prime}:\left(2^{Z}, \preceq_{\phi}^{l}\right) \rightarrow \overline{\mathbb{R}}$ is clearly strictly $\preceq_{\phi}^{l}$-increasing. The rest of the conclusion follows from Lemma 3.1 taking $E:=\mathcal{A}$.

Theorem 3.2 (as well as Theorem 3.3) shows the usefulness of finding a monotone function on a preordered set for proving the existence of minimal (maximal) elements. This will be the main procedure for getting variants of the Ekeland variational principle (EVP) for vector and set-valued functions.

\section{A few technical notions and results}

Throughout this section $X, Y, K, H$ are as in Section 2 (if not stated otherwise explicitly), that is, $(X, d)$ is a metric space, $Y$ is a separated topological vector space, $K \subset Y$ is a proper convex cone, and $H \subset K \backslash(-K)$ is a nonempty $K$-convex set. As seen in Section 2 , ${ }_{H}:=\preceq_{F_{H}}$ is a preorder on $X \times 2^{Y}$. We also consider a set $\mathcal{A} \subseteq X \times 2^{Y}$ such that

$$
Y_{\mathcal{A}}:=\bigcup\left\{A \mid A \in \operatorname{Pr}_{2^{Y}}(\mathcal{A})\right\} \neq \emptyset
$$

where $\operatorname{Pr}_{2^{Y}}(\mathcal{A})=\{B \subseteq Y \mid \exists x \in X:(x, B) \in \mathcal{A}\}$.

Eventually, we are interested in obtaining results similar to the one in Theorem 3.7 this is because, when $Z:=\mathbb{R}$ endowed with the usual order, having $f: X \rightarrow \overline{\mathbb{R}}$ a proper function and $\mathcal{A}:=\left\{\left(x, F_{f}(x)\right) \mid x \in \operatorname{dom} f\right\}$ (where $F_{f}(x):=\{f(x)\}$ for $x \in \operatorname{dom} f, F_{f}(x):=\emptyset$ for $x \in X \backslash \operatorname{dom} f)$ endowed with the preorder $(x,\{f(x)\}) \preceq\left(x^{\prime},\left\{f\left(x^{\prime}\right)\right\}\right)$ defined by $f\left(x^{\prime}\right) \geq$ $f(x)+d\left(x, x^{\prime}\right)$ (or equivalently $F_{f}\left(x^{\prime}\right) \subset F_{f}(x)+d\left(x, x^{\prime}\right)+\mathbb{R}_{+}$) when $x, x^{\prime} \in \operatorname{dom} f$, the conclusion of Theorem 3.7 is saying that for every $x_{0} \in \operatorname{dom} f$ there exists $\bar{x} \in \operatorname{dom} f$ such that $f(\bar{x})+d\left(x_{0}, \bar{x}\right) \leq f\left(x_{0}\right)$ and $f(\bar{x})<f(x)+d(x, \bar{x})$ for $x \in X \backslash\{\bar{x}\}$, that is, the conclusions of one of the usual variants of the Ekeland variational principle (EVP for short). One of the hypotheses of EVP is the lower boundedness of $f$; this is equivalent to each of the following conditions:

$$
\begin{aligned}
& \exists B \subset \mathbb{R} \text { bounded }: F_{f}(X) \subset B+\mathbb{R}_{+}, \\
& \exists B \subset \mathbb{R} \text { bounded, } \forall x \in X: B \not \subset F_{f}(x)+\mathbb{R}_{+}, \\
& \exists a>0, \exists \alpha \in \mathbb{R}, \forall t \in F_{f}(X): \alpha \leq a t .
\end{aligned}
$$

In the $(\varepsilon, \lambda)$ variants of EVP (with $\varepsilon, \lambda>0$ ) one has a fixed $x_{0} \in X$ with $f\left(x_{0}\right) \leq \inf f+\varepsilon$. When using $F_{f}$ this condition is very close to

$$
\forall x \in X: F_{f}\left(x_{0}\right) \not \subset F_{f}(x)+\varepsilon+\mathbb{R}_{+}=F_{f}(x)+\varepsilon \cdot 1+\mathbb{R}_{+} .
$$

The natural extensions of these conditions for a nonempty subset $\mathcal{A}$ of $X \times 2^{Y}$ (in particular for $\Gamma: X \rightrightarrows Y$ with $\operatorname{dom} \Gamma \neq \emptyset$ and $\mathcal{A}:=\{(x, \Gamma(x)) \mid x \in \operatorname{dom} \Gamma\})$ and the nonempty subset 
$H \subset K$ are:

$$
\begin{aligned}
& \exists B \subset Y \text { bounded }: Y_{\mathcal{A}} \subset B+K, \\
& \exists B \subset Y \text { bounded, } \forall A \in Y_{\mathcal{A}}: B \not \subset A+K, \\
& \exists y^{*} \in K^{+} \backslash\{0\}, \exists \alpha \in \mathbb{R}, \forall y \in Y_{\mathcal{A}}: \alpha \leq y^{*}(y), \\
& \forall A \in \operatorname{Pr}_{2^{Y}}(\mathcal{A}): A_{0} \not \subset A+\varepsilon H+K,
\end{aligned}
$$

where $\varepsilon>0$ and $A_{0} \in \operatorname{Pr}_{2} Y(\mathcal{A})$. In the literature, one finds several boundedness notions wrt the convex cone $K \subset Y$. Having the set $E \subseteq Y, E$ is (vector) $K$-bounded from below if there exists $b \in Y$ such that $E \subset b+K ; E$ is quasi $K$-bounded from below if there exists a bounded set $B \subset Y$ such that $E \subset B+K$; $E$ is $K$-bounded if for every neighborhood $U$ of $0 \in Y$ there exists $\lambda>0$ such that $E \subset \lambda U+K ; E$ is $K^{+}$-bounded from below if $y^{*}(E)$ is bounded from below for every $y^{*} \in K^{+}$.

In the following, the "from below" part of the corresponding expression will be dropped if, but we will keep the "(vector)" part in the first notion since it refers to boundedness from below wrt the vector preorder generated by $K$ and in order to avoid confusion with $K$-boundedness.

Among these boundedness notions the implications below hold:

$$
\begin{aligned}
E \text { is (vector) } \mathrm{K} \text {-bounded } & \Rightarrow E \text { is quasi } K \text {-bounded } \\
& \Rightarrow E \text { is } K \text {-bounded } \\
& \Rightarrow E \text { is } K^{+} \text {-bounded; }
\end{aligned}
$$

moreover, if int $K \neq \emptyset$ we have that $E$ is $K$-bounded $\Rightarrow E$ is (vector) $K$-bounded.

The first implication is obvious. The second implication is stated in [20, Lem. 1.3.2]; its converse implication is true when $Y$ is normable as seen in [20, Lem. 1.3.2], too. For the third implication consider $y^{*} \in K^{+}$and take $U:=\left\{y \in Y \mid\left\langle y, y^{*}\right\rangle \geq-1\right\}$. Because $U \in \mathcal{N}_{Y}$, there exists $\lambda>0$ such that $E \subset \lambda U+K$, and so

$$
y^{*}(E) \subset \lambda y^{*}(U)+y^{*}(K) \subset \lambda \cdot[-1, \infty)+[0, \infty)=[-\lambda, \infty) .
$$

Assume now that int $K \neq \emptyset$ and $E$ is $K$-bounded. Take $k^{0} \in \operatorname{int} K$, that is $K-k^{0} \in \mathcal{N}_{Y}$. Then there exists $\lambda>0$ such that $E \subset \lambda\left(K-k^{0}\right)+K=\left(-\lambda k^{0}\right)+K$, whence $E$ is (vector) $K$-bounded.

Example 4.1 It is known that the mapping $\|\cdot\|_{p}$ defined by $\|f\|_{p}:=\left(\int_{(0,1)}|f|^{p} d \lambda\right)^{1 / p}$ (where $\lambda$ is the Lebesgue measure) is a quasinorm on $Y:=L^{p}(0,1)$ for $p \in(0,1)$. So $Y$ is a topological vector space; it is also known that $Y^{*}=\{0\}$, see [16, p. 158, Eq. (9)]. Taking $K:=\left\{f \in L^{p}(0,1) \mid f \geq 0\right.$ a.e. $\}$ we have that $K$ is a closed convex cone with $K^{+}=\{0\}$, and so $Y$ is $K^{+}$-bounded, but clearly, $Y$ is not $K$-bounded.

For a subset $E$ of $Y$ we denote by $\mathrm{cl}_{s e q} E$ the set of those $y \in Y$ such that there exists $\left(y_{n}\right)_{n \geq 1} \subset E$ with $y_{n} \rightarrow y$; we say that $E$ is sequentially closed (seq-closed for short) if $E=\mathrm{cl}_{\text {seq }} E$. Moreover, we say that $E$ is sequentially compact (seq-compact for short) if any sequence from $E$ has a subsequence converging to an element of $E$. Of course, if the topology of $Y$ is metrizable, then $\mathrm{cl}_{\text {seq }} E=\mathrm{cl} E$, and $E$ is seq-compact if and only if $E$ is compact.

In Proposition 4.3 and Example 4.5 (below) we mention several relations among conditions (32) -(35). First we give a preliminary result. 
Lemma 4.2 Let $E \subset Y$ be nonempty, and set $\widetilde{E}:=E \cap(-\mathbb{P} H-K)$, where $\mathbb{P}:=] 0, \infty[$. If one of the following conditions holds:

(i) (a) either $\widetilde{E}$ is quasi $K$-bounded and $0 \notin \mathrm{cl}_{\text {seq }}(H+K)$, or $\widetilde{E}$ is $K$-bounded and $0 \notin \operatorname{cl}(H+K)$;

(ii) there exists $z^{*} \in K^{+}$such that inf $z^{*}(\widetilde{E})>-\infty$ and $\inf z^{*}(H)>0$;

(iii) $\widetilde{E}$ is $K^{+}$-bounded and (18) is satisfied, then

$$
\exists \varepsilon>0: E \cap(-\varepsilon H-K)=\emptyset .
$$

Moreover, if $\left(x_{0}, A_{0}\right) \in \mathcal{A} \subset X \times 2^{Y}, y_{0} \in A_{0}$ and (37) holds for $E:=\left(Y_{\mathcal{A}}-y_{0}\right)$, then (35) holds with $\varepsilon$ provided by (37).

Proof. We prove (i)-(iii) by contradiction. So, assume that (37) does not hold, and so for each $n \in \mathbb{N} \backslash\{0\}$ there exists

$$
y_{n} \in E \cap(-n H-K) \subset E \cap(-\mathbb{P}(H+K))=\widetilde{E}
$$

hence there exist $h_{n} \in H$ and $k_{n} \in K$ such that $y_{n}=-n h_{n}-k_{n}$ (for $n \geq 1$ ). It follows that $\beta_{z^{*}}:=\inf z^{*}(\widetilde{E})<+\infty$ for $z^{*} \in K^{+}$. Moreover,

$$
\beta_{z^{*}} \leq z^{*}\left(y_{n}\right)=-n \cdot z^{*}\left(h_{n}\right)-z^{*}\left(k_{n}\right) \leq-n \cdot z^{*}\left(h_{n}\right) \quad \forall n \geq 1, \forall z^{*} \in K^{+} .
$$

(i) (a) Let $\widetilde{E} \subset B+K$ with $B \subset Y$ a bounded set. Since $y_{n} \in \widetilde{E}$, there exist also $b_{n} \in B$ and $k_{n}^{\prime} \in K$ such that $y_{n}=b_{n}+k_{n}^{\prime}$ for $n \geq 1$. It follows that $-\frac{1}{n} b_{n}=h_{n}+\frac{1}{n}\left(k_{n}+k_{n}^{\prime}\right) \in H+K$ for $n \geq 1$. Since $\left(b_{n}\right)$ is bounded, we get the contradiction $0 \in \operatorname{cl}_{s e q}(H+K)$.

(b) Because $0 \notin \operatorname{cl}(H+K)$, there exists a balanced $U \in \mathcal{N}_{Y}$ such that $U \cap(H+K)=\emptyset$. Since $\widetilde{E}$ is $K$-bounded, there exists $\lambda>0$ such that $\widetilde{E} \subset \lambda U+K$. Since $y_{n} \in \widetilde{E}$, there exists $u_{n} \in U$ and $k_{n}^{\prime} \in K$ such that $y_{n}=\lambda u_{n}+k_{n}^{\prime}$ for $n \geq 1$. It follows that $-\frac{\lambda}{n} u_{n}=$ $h_{n}+\frac{1}{n}\left(k_{n}+k_{n}^{\prime}\right) \in H+K$ for $n \geq 1$. Taking $n \in \mathbb{N}^{*}$ such that $n \geq \lambda$, we obtain the contradiction $-\frac{\lambda}{n} u_{n} \in U \cap(H+K) \neq \emptyset$.

(ii) For $z^{*}$ provided by our assumption we have that $\alpha_{z^{*}}:=\inf z^{*}(H)>0$ and $\beta_{z^{*}} \in \mathbb{R}$. Using (39) we obtain that $\beta_{z^{*}} \leq-n \alpha_{z^{*}}$ for $n \geq 1$. Taking the limit for $n \rightarrow \infty$ we get the contradiction $\beta_{z^{*}}=-\infty$.

(iii) By (18), there exists $z^{*} \in K^{+}$such that $\lim \sup z^{*}\left(h_{n}\right)>0$. From (39) we have that $z^{*}\left(h_{n}\right) \leq-n^{-1} \beta_{z^{*}}$ for $n \geq 1$, whence the contradiction $\lim \sup z^{*}\left(h_{n}\right) \leq 0$.

For the last assertion just observe that, for $E:=\left(Y_{\mathcal{A}}-y_{0}\right)$, (37) is saying that there exists $\varepsilon>0$ such that $y_{0} \notin Y_{\mathcal{A}}+\varepsilon H+K$ which clearly implies (35).

Using Lemma 4.2 (i) one gets [23, Prop. 2.2] taking $E:=\Gamma(X)-y$ with $y \in Y$; there $H=\left\{k_{0}\right\} \subset K \backslash\{0\}, K$ being a pointed closed convex cone. Lemma 4.2 (ii) is obtained in [27, Prop. 5.2]; there $E:=f(X)-f\left(x_{0}\right)$ for some function $f: X \rightarrow Y$.

Proposition 4.3 Let $\mathcal{A} \subset X \times 2^{Y}$ be such that $Y_{\mathcal{A}} \neq \emptyset$.

(i) Assume that either (a) $0 \notin \mathrm{cl}_{\text {seq }}(H+K)$ and $Y_{\mathcal{A}}$ is quasi $K$-bounded, or (b) $0 \notin$ $\operatorname{cl}(H+K)$ and $Y_{\mathcal{A}}$ is $K$-bounded; then

$$
\forall y \in Y, \exists \varepsilon>0: Y_{\mathcal{A}} \cap(y-\varepsilon H-K)=\emptyset .
$$

(ii) Assume that either (a) there exists $z^{*} \in K^{+}$such that inf $z^{*}\left(Y_{\mathcal{A}}\right)>-\infty$ and $\inf z^{*}(H)>$ 0 , or (b) $Y_{\mathcal{A}}$ is $K^{+}$-bounded and (18) is satisfied. Then (40) holds. 
(iii) Assume that $H$ is seq-compact. If there exists a convex cone $C \subset Y$ and a bounded set $B \subset Y$ such that $K \subset C, H \subset \operatorname{int} C$ and $B \not \subset A+C$ for every $A \in \operatorname{Pr}_{2^{Y}}(\mathcal{A})$, then (40) holds; in particular, if $H \subset$ int $K$ and (33) is verified then (40) holds.

(iv) Assume that (40) is verified. Then (33) holds, and for every nonempty set $A_{0} \in$ $\operatorname{Pr}_{2^{Y}}(\mathcal{A})$ there exists $\varepsilon>0$ such that (35) holds.

Proof. (i) (a) Take $y \in Y$ and set $E:=Y_{\mathcal{A}}-y$. Then $E$ is quasi $K$-bounded and $\widetilde{E}:=$ $E \cap(-\mathbb{P}(H+K)) \subset E$, and so $\widetilde{E}$ is quasi $K$-bounded. By Lemma 4.2 (i), there exists $\varepsilon>0$ such that $Y_{\mathcal{A}}-y=E \subset-\varepsilon H-K$, whence the desired conclusion holds. The proof of case (b) is similar.

(ii) Using now Lemma 4.2 (ii) and (iii), the same approach as in (i) shows that (40) holds when (a) or (b) is verified, respectively.

(iii) Assume that (40) does not hold. Then there exists $y_{0} \in Y$ such that for every $n \in \mathbb{N}^{*}$, there exists $A_{n} \in \operatorname{Pr}_{2^{Y}}(\mathcal{A})$ with $A_{n} \cap\left(y_{0}-n H-K\right) \neq \emptyset$; take $y_{n} \in A_{n} \cap\left(y_{0}-n H-K\right)$. Since $B \not \subset A_{n}+C, B \not \subset y_{n}+C$, and so there exists $b_{n} \in B$ such that $b_{n} \notin y_{n}+C$. It follows that $b_{n}-y_{n} \notin C$, whence $b_{n}-y_{n} \in Y \backslash C \subset Y \backslash \operatorname{int} C$. From our choice of $y_{n}$, there exists $h_{n} \in H$ such that $y_{n}-y_{0}+n h_{n} \in-K \subset-C$. It follows that

$$
b_{n}-y_{0}+n h_{n}=\left(b_{n}-y_{n}\right)+\left(y_{n}-y_{0}+n h_{n}\right) \in(Y \backslash \operatorname{int} C)-C \subset Y \backslash \operatorname{int} C .
$$

Since $Y \backslash \operatorname{int} C$ is a cone, we obtain that $\frac{1}{n}\left(b_{n}-y_{0}\right)+h_{n} \in Y \backslash \operatorname{int} C$. Because $H$ is seqcompact, there exists a strictly increasing sequence $\left(n_{p}\right)_{p \geq 1} \subset \mathbb{N}^{*}$ such that $h_{n_{p}} \rightarrow h \in H$. It follows that $\frac{1}{n_{p}}\left(b_{n_{p}}-y_{0}\right)+h_{n_{p}} \rightarrow h \in \mathrm{cl}_{\text {seq }}(Y \backslash \operatorname{int} C)=Y \backslash \operatorname{int} C$, contradicting the fact that $H \subset \operatorname{int} C$.

(iv) For the first assertion, taking $y:=0$ in (40), there exists $\varepsilon>0$ such that $Y_{\mathcal{A}} \cap(-\varepsilon H-$ $K)=\emptyset$. Hence (33) holds for $B:=\left\{-\varepsilon h^{0}\right\}$ with $h^{0} \in H$.

For the second assertion, take $A_{0} \in \operatorname{Pr}_{2^{Y}}(\mathcal{A})$ with $A_{0} \neq \emptyset$. Consider $y_{0} \in A_{0}$. Since (40) holds, there exists $\varepsilon>0$ such that $Y_{\mathcal{A}} \cap\left(y_{0}-\varepsilon H-K\right)=\emptyset$, that is $y_{0} \notin Y_{\mathcal{A}}+\varepsilon H+K$. Hence $A_{0} \not \subset Y_{\mathcal{A}}+\varepsilon H+K$, and so $A_{0} \not \subset A+\varepsilon H+K$ for any $A \in \operatorname{Pr}_{2^{Y}}(\mathcal{A})$. The proof is complete.

Some relations among the conditions on $H$ used in Proposition 4.3 are provided in the next result.

Lemma 4.4 (i) If $\emptyset \neq S, T \subset Y$ and $S$ is seq-compact then $\operatorname{cl}_{\text {seq }}(S+T)=S+\operatorname{cl}_{\text {seq }} T$; in particular, if $H$ is seq-compact, then $0 \notin \mathrm{cl}_{\text {seq }}(H+K)$ if and only if $H \cap\left(-\mathrm{cl}_{\text {seq }} K\right)=\emptyset$.

(ii) The following implications hold:

$$
\left[\exists z^{*} \in K^{+}: \inf z^{*}(H)>0\right] \Rightarrow(\underline{18}) \Rightarrow 0 \notin \mathrm{cl}_{\text {seq }}(H+K) .
$$

(iii) Assume that $H$ is seq-compact and $C \subset Y$ is a proper convex cone such that $K \subset C$ and $H \subset \operatorname{int} C$. Then $0 \notin \mathrm{cl}_{\text {seq }}(H+K)$.

Proof. (i) The inclusion $S+\mathrm{cl}_{\text {seq }} T \subset \mathrm{cl}_{\text {seq }}(S+T)$ is obvious. Assume that $S$ is seqcompact and take $y \in \operatorname{cl}_{\text {seq }}(S+T)$. Then there exist $\left(u_{n}\right)_{n \geq 1} \subset S$ and $\left(v_{n}\right)_{n \geq 1} \subset T$ such that $u_{n}+v_{n} \rightarrow y$. Because $S$ is seq-compact, there exists a strictly increasing sequence $\left(n_{p}\right)_{p \geq 1} \subset \mathbb{N}^{*}$ such that $u_{n_{p}} \rightarrow u \in S$. It follows that $v_{n_{p}}=\left(u_{n_{p}}+u_{n_{p}}\right)-u_{n_{p}} \rightarrow v:=y-u$, and so $y=u+v \in S+\operatorname{cl}_{s e q} T$. 
(ii) The first implication in (41) is obvious. Assume that $0 \in \mathrm{cl}_{\text {seq }}(H+K)$ and take $z^{*} \in K^{+}$. Then there exists $\left(h_{n}\right)_{n \geq 1} \subset H$ and $\left(k_{n}\right)_{n \geq 1} \subset K$ such that $y_{n}:=h_{n}+k_{n} \rightarrow 0$. Since $z^{*}\left(y_{n}\right)=z^{*}\left(h_{n}\right)+z^{*}\left(k_{n}\right) \geq z^{*}\left(h_{n}\right) \geq 0$, it follows that $z^{*}\left(h_{n}\right) \rightarrow 0$. This shows that (18) does not hold. Hence, the second implication (41) holds, too.

(iii) Clearly, $\mathrm{cl}_{\text {seq }} K \subset \mathrm{cl} C$, and so, using (i) and the properness of $C$,

$$
\mathrm{cl}_{\text {seq }}(H+K)=H+\mathrm{cl}_{\text {seq }} K \subset \operatorname{int} C+\operatorname{cl} C=\operatorname{int} C .
$$

Since $0 \notin \operatorname{int} C$ ( $C$ being proper $), 0 \notin \mathrm{cl}_{\text {seq }}(H+K)$.

The example below shows that some of the reversed implications in Proposition 4.3 are not valid.

Example 4.5 Let $Y:=\mathbb{R}^{2}$ be endowed with the Euclidean norm, $K:=\mathbb{R}_{+}^{2}, H:=\left\{k^{0}\right\}$ with $k^{0}:=(1,0) \in K$ and $E:=\{0\} \times \mathbb{R}_{-}$, where $\mathbb{R}_{-}:=-\mathbb{R}_{+}$. It is clear that $H$ is compact, $E$ is not $K^{+}$-bounded (for example $\varphi: \mathbb{R}^{2} \rightarrow \mathbb{R}$ defined by $\varphi\left(y_{1}, y_{2}\right):=y_{2}$ is in $K^{+}$and $\inf \varphi(E)=-\infty)$, and so, by (36), $E$ is not quasi $K$-bounded. However, (40) holds for $Y_{\mathcal{A}} \subset E$. Indeed, for $y=\left(y_{1}, y_{2}\right) \in \mathbb{R}^{2}$, take $\varepsilon:=1+\max \left\{0, y_{1}\right\}$; then $E \cap\left(y-\varepsilon k^{0}-K\right)=\emptyset$.

The following result will play an important role in the next sections.

Proposition 4.6 Assume that $\left(\left(x_{n}, A_{n}\right)\right)_{n \geq 1} \subset X \times 2^{Y}$ is a $\preceq_{H}$-decreasing sequence with $A_{1} \neq \emptyset$. Set $E:=\cup_{n \geq 1} A_{n}$, and consider the following assertions:

(i) either (a) $0 \notin \mathrm{cl}_{\text {seq }}(H+K)$ and $E$ is quasi $K$-bounded, or (b) $0 \notin \operatorname{cl}(H+K)$ and $E$ is $K$-bounded;

(ii) there exists $z^{*} \in K^{+}$such that $\inf z^{*}(E)>-\infty$ and $\inf z^{*}(H)>0$;

(iii) the set $E$ is $K^{+}$-bounded and condition (18) is satisfied;

(iv) $H$ is seq-compact, and there exist a bounded set $B \subset Y$ and a convex cone $C$ such that $K \subset C, H \subset \operatorname{int} C$ and $B \not \subset A_{n}+C$ for $n \geq 1$;

(v) there exist $\alpha>0$ and an infinite set $P \subset \mathbb{N}^{*}$ such that $A_{1} \not \subset\left(A_{n}+\alpha H+K\right)$ for all $n \in P$.

Then [(i) $\vee$ (ii) $\vee$ (iii) $\vee($ iv $)] \Rightarrow(\mathrm{v}) \Rightarrow \sum_{n \geq 1} d\left(x_{n}, x_{n+1}\right) \leq \alpha$; in particular, if at least one of the assertions (i)-(v) holds, then $\left(x_{n}\right)_{n \geq 1}$ is a Cauchy sequence.

Proof. Assume that (i), or (ii), or (iii), or (iv) holds. Taking $\mathcal{A}:=\left\{\left(x_{n}, A_{n}\right) \mid n \in \mathbb{N} \backslash\{0\}\right\}$ and using Proposition 4.3 (i), (ii)(a), (ii)(b) or (iii), respectively, we obtain that (40) holds. Applying now Proposition 4.3 (iv), we obtain that (v) holds.

Assume that (v) holds. Since $\left(x_{n+1}, A_{n+1}\right) \preceq_{H}^{1}\left(x_{n}, A_{n}\right)$ for $n \geq 1$, we have that

$$
A_{n} \subset A_{n+1}+d\left(x_{n}, x_{n+1}\right) H+K \quad \forall n \geq 1 .
$$

Using (16) and the inclusions in (42), we obtain that

$$
A_{1} \subset A_{n+1}+\mu_{n} H+K \quad \forall n \geq 1,
$$

where $\mu_{n}:=\sum_{l=1}^{n} d\left(x_{l}, x_{l+1}\right)$. Since $A_{1} \not \subset A_{n+1}+\alpha H+K$ for $n+1 \in P$, we have that $\mu_{n}<\alpha$; otherwise (that is $\mu_{n} \geq \alpha$ ), from (43) we get the contradiction

$$
A_{1} \subset A_{n+1}+\mu_{n} H+K \subset A_{n+1}+\left(\mu_{n}-\alpha\right) H+\alpha H+K \subset A_{n+1}+\alpha H+K .
$$

The set $P$ being infinite, $\mu_{n}<\alpha$ for every $n \geq 1$, and so $\sum_{n \geq 1} d\left(x_{n}, x_{n+1}\right)=\lim \mu_{n} \leq \alpha$. 
Corollary 4.7 Let $\mathcal{A} \subset X \times 2^{Y}$ be such that $A \neq \emptyset$ for every $A \in \operatorname{Pr}_{2^{Y}}(\mathcal{A})$. Then $\left(\mathcal{A}, \preceq_{H}\right)$ verifies condition $(\mathrm{Ca})$ whenever one of the following assertions holds:

(i) either (a) $0 \notin \mathrm{cl}_{\text {seq }}(H+K)$ and $Y_{\mathcal{A}}$ is quasi $K$-bounded, or (b) $0 \notin \mathrm{cl}(H+K)$ and $Y_{\mathcal{A}}$ is $K$-bounded;

(ii) there exists $z^{*} \in K^{+}$such that $\inf z^{*}\left(Y_{\mathcal{A}}\right)>-\infty$ and $\inf z^{*}(H)>0$;

(iii) $Y_{\mathcal{A}}$ is $K^{+}$-bounded and condition (18) is satisfied;

(iv) $H$ is seq-compact, and there exist a bounded set $B \subset Y$ and a convex cone $C$ such that $K \subset C, H \subset \operatorname{int} C$ and $B \not \subset A+C$ for every $A \in \operatorname{Pr}_{2^{Y}}(\mathcal{A})$.

Proof. It is an immediate consequence of Propositions 4.3 and 4.6 ,

In the next section we shall use also cs-complete sets. Recall that a set $E \subseteq Y$ is $c s$ complete (see [32, p. 9]) if for all sequences $\left(\lambda_{n}\right)_{n \geq 1} \subseteq \mathbb{R}_{+},\left(y_{n}\right)_{n \geq 1} \subseteq E$ such that $\sum_{n \geq 1} \lambda_{n}=1$ and the sequence $\left(\sum_{m=1}^{n} \lambda_{m} y_{m}\right)_{n>1}$ is Cauchy, the series $\sum_{n>1} \lambda_{n} y_{n}$ (called convex series with elements of $E$ ) is convergent and its sum belongs to $E$. One says that $E \subseteq Y$ is cs-closed if the sum of any convergent convex series with elements from $E$ belongs to $E$. Of course, any cs-complete set is cs-closed; if $Y$ is (sequentially) complete then the converse is true. Notice also that any cs-closed set is convex. Moreover, it is worth observing that the closed convex subsets of topological vector spaces are cs-closed, as well as the open convex subsets of separated locally convex spaces; furthermore, all the convex subsets of finite dimensional normed spaces are cs-closed (hence cs-complete).

Notice that $E \subset Y$ is cs-complete if and only if for all sequences $\left(\lambda_{n}\right)_{n \geq 1} \subseteq \mathbb{R}_{+},\left(y_{n}\right)_{n \geq 1} \subseteq$ $E$ such that $\lambda:=\sum_{n \geq 1} \lambda_{n} \in \mathbb{R}_{+}^{*}$ and the sequence $\left(\sum_{m=1}^{n} \lambda_{m} y_{m}\right)_{n \geq 1}$ is Cauchy, the series $\sum_{n \geq 1} \lambda_{n} y_{n}$ converges to some $y \in Y$ and $\lambda^{-1} y \in E$. Similarly, $E$ is cs-closed if and only if for all sequences $\left(\lambda_{n}\right)_{n \geq 1} \subseteq \mathbb{R}_{+},\left(y_{n}\right)_{n \geq 1} \subseteq E$ such that $\lambda:=\sum_{n \geq 1} \lambda_{n} \in \mathbb{R}_{+}^{*}$ and $y:=$ $\sum_{n \geq 1} \lambda_{n} y_{n} \in Y$, one has $\lambda^{-1} y \in E$. Note that in [21, Def. 2.1.4] it is said that $E$ is $\sigma$-convex or cs-compact if any convex series with elements from $E$ is convergent and its sum belongs to $E$. It is quite easy to prove that $E \subset Y$ is $\sigma$-convex if and only if $E$ is cs-complete and bounded; this assertion is formulated in [27, p. 19] (see also [25, p. 921], where it is asserted that $E$ is $\sigma$-convex if and only if $E$ is cs-complete).

Proposition 4.8 Assume that $E \subset Y$ is a nonempty seq-compact convex set. Then $E$ is cs-complete.

Proof. Since $E$ is seq-compact, $E$ is bounded. Take $\left(\lambda_{n}\right)_{n \geq 1} \subset \mathbb{R}_{+}^{*}$ with $\sum_{n \geq 1} \lambda_{n}=1$ and $\left(v_{n}\right)_{n \geq 1} \subset E$. For this, fix $V \subseteq Y$ a balanced neighborhood of 0 . Because $E$ is bounded, there exists $\alpha>0$ such that $E \subseteq \alpha V$. Since the series $\sum_{n \geq 1} \lambda_{n}$ is convergent there exists $n_{0} \geq 1$ such that $\sum_{k=n}^{n+p} \lambda_{k} \leq \alpha^{-1}$ for all $n, p \in \mathbb{N}$ with $n \geq n_{0}$. Since $E$ is convex, for such $n, p$ there exists $v_{n, p} \in E$ with

$$
\sum_{k=n}^{n+p} \lambda_{k} v_{k}=\left(\sum_{k=n}^{n+p} \lambda_{k}\right) v_{n, p} \in\left[0, \alpha^{-1}\right] E \subseteq\left[0, \alpha^{-1}\right] \alpha V=V ;
$$

hence $\left(y_{n}\right)_{n \geq 1}$ is a Cauchy sequence, where $y_{n}:=\sum_{m=1}^{n} \lambda_{m} v_{m}$ for $n \geq 1$. Set also $\mu_{n}:=$ $\sum_{m=1}^{n} \lambda_{m} \in[0,1]$. Since $\mu_{n} \rightarrow 1$, we may (and do) assume that $\mu_{n}>0$ for $n \geq 1$. Since $E$ is convex, $v_{n}^{\prime}:=\mu_{n}^{-1} y_{n} \in E$, while because $E$ is seq-compact, $\left(v_{n}^{\prime}\right)$ has a subnet converging to $v \in E$. Because $\mu_{n} \rightarrow 1$, the corresponding subnet of $\left(y_{n}\right)$ converges to $v$. Since $\left(y_{n}\right)$ is a Cauchy sequence, $y_{n} \rightarrow v$. Hence $E$ is cs-complete.

Clearly, the converse of the preceding result is not true in general; for example, any nonempty open convex subset of a Banach space is cs-complete (but, clearly, is not compact). 


\section{Ekeland's Variational Principles of Ha-Hamel-Löhne's Type}

Throughout this section $X, Y, K, F$ are as in Section 2 (if not stated otherwise explicitly), that is, $(X, d)$ is a metric space, $Y$ is a separated topological vector space, $K \subset Y$ is a proper convex cone, and $F: X \times X \rightrightarrows K$ verifies conditions (F1) and (F2). On $X \times 2^{Y}$ we consider the preorder $\preceq_{F}$, as well as $\preceq_{F, z^{*}}$ for $z^{*} \in K^{+}$, defined in (3) and (23), respectively. Taking into account that $(x, A) \preceq_{F}\left(x^{\prime}, \emptyset\right)$ for all $x, x^{\prime} \in X$ and $A \in 2^{Y}$, in the sequel we assume that

(H) $\mathcal{A} \subseteq X \times 2^{Y}$ is nonempty and $A \neq \emptyset$ for every $A \in \operatorname{Pr}_{2^{Y}}(\mathcal{A})$.

Hence

$$
Y_{\mathcal{A}}:=\bigcup\left\{A \mid A \in \operatorname{Pr}_{2^{Y}}(\mathcal{A})\right\} \neq \emptyset .
$$

An important example of set $\mathcal{A} \subseteq X \times 2^{Y}$ which will be considered often in the sequel is

$$
\mathcal{A}_{\Gamma}:=\{((x, \Gamma(x))) \mid x \in \operatorname{dom} \Gamma\},
$$

where $\Gamma: X \rightrightarrows Y$ with $\operatorname{dom} \Gamma \neq \emptyset$; of course, $Y_{\mathcal{A}_{\Gamma}}=\Gamma(X)$. Ha [8] established an EVP type result on a complete metric space $X$ for a set-valued function $\Gamma: X \rightrightarrows Y$ which uses the relation $\leq_{K}^{l}$ (see Section 2) for comparing the values of $\Gamma$; Hamel [9] and Hamel-Löhne 12] established more general results for subsets $\mathcal{A} \subseteq X \times 2^{Y}$ even for a uniform space $X$. Corollary 5.14 below is an extension of this type of results.

The first part of the next result is a translation of Theorem 3.7 to the present context. For getting the second part we use Lemma 3.1 and Theorem 3.2 .

Theorem 5.1 Assume that $\left(\mathcal{A}, \preceq_{F}\right)$ verifies condition $(\mathrm{C} 0)$. Then:

(i) for every $(x, A) \in \mathcal{A}$ there exists $(\bar{x}, \bar{A}) \in \mathcal{A}$ such that $(\bar{x}, \bar{A}) \preceq_{F}(x, A)$ and $\mathcal{A} \ni$ $\left(x^{\prime}, A^{\prime}\right) \preceq_{F}(\bar{x}, \bar{A})$ implies $x^{\prime}=\bar{x}$;

(ii) assume that $z^{*} \in K^{+}$is such that inf $z^{*}\left(F\left(x, x^{\prime}\right)\right)>0$ for $x, x^{\prime} \in X$ with $x \neq x^{\prime}$ and $\inf z^{*}(A)>-\infty$ for $A \in \operatorname{Pr}_{2^{Y}}(\mathcal{A})$; then for every $(x, A) \in \mathcal{A}$ there exists $(\bar{x}, \bar{A}) \in \mathcal{A}$ minimal wrt $\preceq_{F, z^{*}}$ such that $(\bar{x}, \bar{A}) \preceq_{F, z^{*}}(x, A)$ and $\mathcal{A} \ni\left(x^{\prime}, A^{\prime}\right) \preceq_{F}(\bar{x}, \bar{A})$ implies $x^{\prime}=\bar{x}$.

Proof. (ii) Take $z^{*} \in K^{+}$provided by the hypothesis. It is clear that $(W, \preccurlyeq):=\left(\mathcal{A}, \preceq_{F}\right)$ is a preordered set. Moreover, using the estimate in (20) we obtain that

$$
\phi: \mathcal{A} \rightarrow \overline{\mathbb{R}}, \quad \phi(x, A):=\inf z^{*}(A),
$$

is $\preceq_{F}$-increasing and real-valued. Clearly, $\preccurlyeq_{\phi}$ from (25) is nothing else than $\preceq_{F, z^{*}}$. Because $\left(\mathcal{A}, \preceq_{F}\right)$ verifies condition (C0), using Proposition 3.5 and Lemma 3.1 (iii), $\left(W, \preccurlyeq_{\phi}\right)$ verifies condition $(\mathrm{Ab})$.

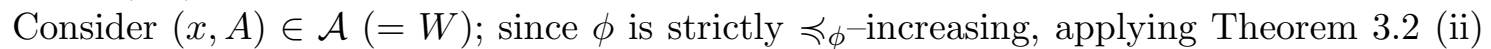
for $\left(W, \preccurlyeq_{\phi}\right)$, we get $(\bar{x}, \bar{A}) \in \mathcal{A}$ minimal wrt $\preceq_{\phi}=\preceq_{F, z^{*}}$ such that $(\bar{x}, \bar{A}) \preceq_{F, z^{*}}(x, A)$. Take $\mathcal{A} \ni\left(x^{\prime}, A^{\prime}\right) \preceq_{F}(\bar{x}, \bar{A})$, that is $W \ni\left(x^{\prime}, A^{\prime}\right) \preceq_{\phi}(\bar{x}, \bar{A})$. Using Lemma 3.1 (ii) we obtain that $\inf z^{*}\left(A^{\prime}\right)=\inf z^{*}(\bar{A})$. Assuming that $x^{\prime} \neq \bar{x}$, we get the contradiction inf $z^{*}(\bar{A}) \geq$ $\inf z^{*}\left(A^{\prime}\right)+\inf z^{*}\left(F\left(x^{\prime}, \bar{x}\right)\right)>\inf z^{*}\left(A^{\prime}\right)$ using (19). The proof is complete.

Remark 5.1 Note that, for having the conclusions of Theorem 5.1 (i) or (ii) only for a given $(x, A) \in \mathcal{A}$, it is sufficient to assume that $(\mathrm{C} 0)$ is verified by the sets

$$
\begin{aligned}
\mathcal{A}_{F}(x, A) & :=\left\{\left(x^{\prime}, A^{\prime}\right) \in \mathcal{A} \mid\left(x^{\prime}, A^{\prime}\right) \preceq_{F}(x, A)\right\}, \\
\mathcal{A}_{F, z^{*}}(x, A) & :=\left\{\left(x^{\prime}, A^{\prime}\right) \in \mathcal{A} \mid\left(x^{\prime}, A^{\prime}\right) \preceq_{F, z^{*}}(x, A)\right\},
\end{aligned}
$$

respectively. 
Remark 5.2 Taking $F\left(x, x^{\prime}\right):=\left\{d\left(x, x^{\prime}\right) k^{0}\right\}$ with $k^{0} \in K \backslash(-\operatorname{cl} K)$ in Theorem 5.1 (using also Remark 5.1) one obtains [12, Th. 5.1] in the case $X$ is a metric space. Indeed, on $\mathcal{A}_{0}$, if (M2) or (M2') is verified, then (i) or (iv) from Proposition 4.6 holds, respectively, and so any $\preceq_{F}$-decreasing sequence in $\mathcal{A}_{0}$ is Cauchy. This together with (M3) shows that (C0) holds. In a similar way [12, Th. 6.1] can be obtained.

In the sequel, the conditions $(\mathrm{Cx})$ or $(\mathrm{Cxx})$ will refer to $\left(\mathcal{A}, \preceq_{F}\right)$. Note that condition (C'1) for $\left(\mathcal{A}, \preceq_{F}\right)$ corresponds to condition (H1) in [29] and [15].

Remark 5.3 Taking $X, Y, K, H$ and $\Gamma: X \rightrightarrows Y$ defined in Example 5.2 below, $\left(\mathcal{A}_{\Gamma}, \preceq_{F}\right)$ verifies conditions $\left(\mathrm{C}^{\prime} 1\right)$ and $(\mathrm{C} 1)$ for $F:=F_{H}$, but not $(\mathrm{C} 0)$. Moreover, the conclusion of Theorem 5.1 (i) does not hold. This shows that, in order to have the conclusion of Theorem 5.1 we need supplementary conditions besides (C'1) or (C1).

Example 5.2 Let $X:=\mathbb{R}$ and $Y:=\mathbb{R}^{2}$ be endowed with their usual norms, $K:=\mathbb{R} \times \mathbb{R}_{+}$, $H:=\left\{\left(y_{1}, y_{2}\right) \in \mathbb{R}_{+}^{2} \mid y_{1} y_{2} \geq 1\right\}$, and $\Gamma: X \rightrightarrows Y, \Gamma(x):=\left\{\left(x, e^{x}\right)\right\}$. It is clear that $H$ is a closed convex subset of $K \backslash\{(0,0)\}$ and $K+\varepsilon H=\operatorname{int} K=\mathbb{R} \times \mathbb{R}_{+}^{*}$ for $\varepsilon>0$, where $\mathbb{R}_{+}^{*}:=\mathbb{R}_{+} \backslash\{0\}$. One has $\Gamma(x)+K=\mathbb{R} \times\left[e^{x}, \infty\right)$, and so, for $x, x^{\prime} \in X$ and $\alpha>0$,

$$
\Gamma(x) \preceq_{H} \Gamma\left(x^{\prime}\right) \Leftrightarrow x \leq x^{\prime} \Leftrightarrow \Gamma(x) \leq_{K}^{l} \Gamma\left(x^{\prime}\right), \quad \Gamma\left(x^{\prime}\right) \subset \Gamma(x)+\alpha H+K \Leftrightarrow x<x^{\prime} ;
$$

moreover, $\Gamma(X)=\left\{\left(x, e^{x}\right) \mid x \in \mathbb{R}\right\} \subset K$, which shows that $\Gamma(X)$ is (vector) $K$-bounded. So, for the sequence $\left(x_{n}\right)_{n \geq 1} \subset X$ with $x_{n} \rightarrow x$ and $\Gamma\left(x_{n}\right) \subset \Gamma\left(x_{n+1}\right)+K$ (that is $\Gamma\left(x_{n+1}\right) \leq_{K}^{l}$ $\left.\Gamma\left(x_{n}\right)\right)$ for $n \geq 1$, we have that $x_{n+1} \leq x_{n}$, and so $x \leq x_{n}$ for $n \geq 1$, whence $\Gamma\left(x_{n}\right) \subset \Gamma(x)+K$ for $n \geq 1$. This shows that (C'1) and (C1) are verified; however, taking $x_{n}:=-n$ (for $n \geq 1$ ) it is clear that $(\mathrm{C} 0)$ is not verified.

Remark 5.4 Let $\Gamma: X \rightrightarrows Y$ have nonempty domain. Set $x \preceq u$ if $(x, \Gamma(x)) \preceq_{F}(u, \Gamma(u))$. Using Remark 3.3, $\left(\mathcal{A}_{\Gamma}, \preceq_{F}\right)$ verifies condition (C'1) if and only if $S(u):=\{x \in X \mid x \preceq u\}$ is $\preceq$-lower closed for every $u \in X$, if and only if $(X, d, \preceq)$ verifies (A'1). This shows that condition (C'1) extends the dynamic closedness of a set-valued mapping as defined in [25] and elsewhere. Also notice that $\left(\mathcal{A}_{\Gamma}, \preceq_{F}\right)$ verifies condition (Ca) if and only if $(X, d, \preceq)$ verifies (Aa).

Lemma 5.3 Assume that $F$ verifies condition (F3), and $z_{F}^{*}\left(Y_{\mathcal{A}}\right)$ is bounded from below, where $z_{F}^{*}$ is provided by $(\mathrm{F} 3)$. Then $\left(\mathcal{A}, \preceq_{F}\right)$ verifies condition (Ca).

Proof. Consider a $\preceq_{F}$-decreasing sequence $\left(\left(x_{n}, A_{n}\right)\right)_{n \geq 1} \subset \mathcal{A}$. Setting $\gamma_{n}:=\inf z^{*}\left(A_{n}\right)$ $(\in \mathbb{R})$, then clearly the sequence $\left(\gamma_{n}\right)_{n \geq 1} \subset \mathbb{R}$ is decreasing and bounded, and so $\gamma:=$ $\lim _{n \rightarrow \infty} \gamma_{n} \in \mathbb{R}$. Using (20), we get

$$
\gamma_{n}-\gamma_{n+1}=\inf z^{*}\left(A_{n}\right)-\inf z^{*}\left(A_{n+1}\right) \geq \inf z^{*}\left(F\left(x_{n+1}, x_{n}\right)\right) \geq \eta\left(d\left(x_{n}, x_{n+1}\right)\right) \quad \forall n \geq 1 .
$$

It follows that $\eta\left(d\left(x_{n}, x_{n+1}\right)\right) \rightarrow 0$, and so $d\left(x_{n}, x_{n+1}\right) \rightarrow 0$ because $\eta: \mathbb{R}_{+} \rightarrow \overline{\mathbb{R}}_{+}$is increasing. Therefore, $\left(x_{n}\right)_{n \geq 1}$ is asymptotic, and so, by Lemma 3.8, (Ca) is verified.

Theorem 5.4 Assume that the following two conditions hold:

(i) F verifies condition (F3),

(ii) $\left(\mathcal{A}, \preceq_{F}\right)$ verifies $(\mathrm{C} 1)$ and $z_{F}^{*}\left(Y_{\mathcal{A}}\right)$ is bounded from below, where $z_{F}^{*}$ is provided by (F3).

Then for every $(x, A) \in \mathcal{A}$ there exists a minimal element $(\bar{x}, \bar{A}) \in \mathcal{A}$ wrt $\preceq_{F, z_{F}^{*}}$ such that $(\bar{x}, \bar{A}) \preceq_{F, z_{F}^{*}}(x, A)$; moreover $\mathcal{A} \ni\left(x^{\prime}, A^{\prime}\right) \preceq_{F}(\bar{x}, \bar{A})$ implies $x^{\prime}=\bar{x}$. 
Proof. Taking into account (9), inf $z_{F}^{*}\left(F\left(x, x^{\prime}\right)\right) \geq \eta\left(d\left(x, x^{\prime}\right)\right)>0$ for $x, x^{\prime} \in X$ with $x \neq x^{\prime}$. Moreover, $\inf z_{F}^{*}(A) \geq \inf z_{F}^{*}\left(Y_{\mathcal{A}}\right)>-\infty$ for every $A \in \operatorname{Pr}_{2^{Y}}(\mathcal{A})$. On the other hand, applying Lemma 5.3, (Ca) is verified. Since (C1) holds, using now Proposition 3.5 we obtain that (C0) is verified. The conclusion follows using Theorem 5.1 (ii).

Note that condition (F2) in Theorem [5.4 was used only for obtaining the transitivity of $\preceq_{F}$; for $G: X \times X \rightrightarrows K$ verifying $(\mathrm{F} 1)$ and $\mathcal{A} \subset X \times 2^{Y}$ we introduce the relation $\preceq_{G}^{\prime}$ on $\mathcal{A}$ defined by $\left(x_{1}, A_{1}\right) \preceq_{G}^{\prime}\left(x_{2}, A_{2}\right)$ if $A_{2} \subset A_{1}+G\left(x_{1}, x_{2}\right)$. Of course, for $z^{*} \in K^{+}$, the relation $\preceq_{G, z^{*}}^{\prime}$ on $\mathcal{A}$ is introduced as in (23) by replacing $\preceq_{F}$ with $\preceq_{G}^{\prime}$. So, we get the following variant of Theorem 5.4 (with the same proof).

Theorem 5.5 Assume that the following two conditions hold:

(i) $G: X \times X \rightrightarrows K$ is such that (F1), (F3) hold, and $\preceq_{G}^{\prime}$ (defined above) is transitive;

(ii) $\mathcal{A}$ verifies $(\mathrm{C} 1)$ with $\preceq_{G}^{\prime}$ instead of $\preceq_{G}$, and $z_{G}^{*}\left(Y_{\mathcal{A}}\right)$ is bounded from below, where $z_{G}^{*}$ is provided by (F3).

Then, for every $(x, A) \in \mathcal{A}$ there exists a minimal element $(\bar{x}, \bar{A}) \in \mathcal{A}$ wrt $\preceq_{G, z_{G}^{*}}^{\prime}$ such that $(\bar{x}, \bar{A}) \preceq_{G, z_{G}^{*}}^{\prime}(x, A) ;$ moreover $\mathcal{A} \ni\left(x^{\prime}, A^{\prime}\right) \preceq_{G}^{\prime}(\bar{x}, \bar{A})$ implies $x^{\prime}=\bar{x}$.

The next result is [29, Th. 4.1] and [15, Th. 10.4.4].

Corollary 5.6 Assume that $(X, d)$ is complete, $F$ satisfies condition (F3) and $\Gamma: X \rightrightarrows Y$ is such that $z_{F}^{*}$ [provided by (F3)] is bounded from below on $\Gamma(X)$ (assumed to be nonempty). If $S(u):=\{x \in X \mid \Gamma(u) \subseteq \Gamma(x)+F(x, u)+K\}$ is closed for every $u \in X$, then for every $x \in \operatorname{dom} \Gamma$ there exists $\bar{x} \in S(x)$ such that $S(\bar{x})=\{\bar{x}\}$.

Proof. Using Lemma 5.3, we have that $\left(\mathcal{A}_{\Gamma}, \preceq_{F}\right)$ verifies condition $(\mathrm{Ca})$, and so $(X, d, \preceq)$ satisfies condition (Aa). As seen in Remark 5.4, condition (A'1) is verified. The conclusion follows using Remark 3.3 and Corollary 3.12 .

Remark 5.5 (i) Taking into account Remark 5.4, instead of assuming in Corollary 5.6 that $S(u)$ is closed for every $u \in X$ it is sufficient to have that $S(u)$ is $\preccurlyeq$-lower closed [that is, $(X, d, \preceq)$ verifies (A'1), or equivalently $\left(\mathcal{A}_{\Gamma}, \preccurlyeq_{F}\right)$ verifies (C'1)], where $\preccurlyeq$ is defined by $x \preccurlyeq u: \Longleftrightarrow x \in S(u)$; moreover, instead of assuming that $(X, d)$ is complete, it is sufficient to suppose that any $\preceq$-decreasing Cauchy sequence is convergent [that is, $(X, d, \preceq)$ verifies (Aa1), or equivalently $\left(\mathcal{A}_{\Gamma}, \preccurlyeq_{F}\right)$ verifies (Ca1)].

(ii) Observe that Theorem 5.4 extends [25, Cor. 3.4] in the case in which $\Lambda$ is a singleton because condition (C'1) is verified when $(X, d)$ is complete and the sets $S(x)$ are dynamically closed for $x \in S\left(x_{0}\right)$ (assumed to be nonempty).

With a similar proof to that of Corollary 5.6 one gets the next result; for $Y$ a separated locally convex space, $K \subseteq Y$ a closed convex cone, $k^{0} \in K \backslash\{0\}, F\left(x, x^{\prime}\right):=\left\{d\left(x, x^{\prime}\right) k^{0}\right\}$ for $x, x^{\prime} \in X$, and $\Gamma(X)$ quasi $K$-bounded, this reduces to [18, Cor. 3.1].

Corollary 5.7 Assume that $(X, d)$ is complete, $F: X \times X \rightrightarrows K$ satisfies conditions (F1) and (F3), and $\Gamma: X \rightrightarrows Y$ is such that $z_{F}^{*}$ (from (F3)) is bounded from below on $\Gamma(X)$. Assume also that (a) $\{x \in X \mid \Gamma(u) \subseteq \Gamma(x)+F(x, u)\}$ is closed for every $u \in X$, and (b) $\Gamma(y) \subseteq \Gamma(x)+F(x, y), \Gamma(z) \subseteq \Gamma(y)+F(y, z)$ imply $\Gamma(z) \subseteq \Gamma(x)+F(x, z)$ for all $x, y, z \in$ $X$. Then for every $x \in \operatorname{dom} \Gamma$ there exists $\bar{x} \in X$ such that $\Gamma(x) \subseteq \Gamma(\bar{x})+F(\bar{x}, x)$, and $\Gamma(\bar{x}) \subseteq \Gamma(x)+F(x, \bar{x})+K$ implies $x=\bar{x}$. 
In the sequel $H \subset K \backslash(-K)$ is a $K$-convex set and $F_{H}$ is defined by (17). As mentioned in Lemma 2.1, $F_{H}$ verifies conditions (F1) and (F2). Of course, any of the preceding results can be reformulated for $F:=F_{H}, \preceq_{H}:=\preceq_{F_{H}}$ and $\preceq_{H, z^{*}}:=\preceq_{F_{H}, z^{*}}$. For example, the version of Theorem 5.4 is the one corresponding to (ii) in the following result. The other situations are more specific to the case $F=F_{H}$.

In the sequel all the conditions and results refer to $\preceq_{H}$.

Theorem 5.8 Let $\left(\mathcal{A}, \preceq_{H}\right)$ verify condition $(\mathrm{C} 1)$ wrt $\preceq_{H}$. Assume that one of the following conditions are verified:

(i) either (a) $0 \notin \mathrm{cl}_{\text {seq }}(H+K)$ and $Y_{\mathcal{A}}$ is quasi $K$-bounded, or (b) $0 \notin \mathrm{cl}(H+K)$ and $Y_{\mathcal{A}}$ is $K$-bounded;

(ii) there exists $z^{*} \in K^{+}$such that $\inf z^{*}(H)>0$ and $\inf z^{*}\left(Y_{\mathcal{A}}\right)>-\infty$,

(iii) $H$ satisfies condition (18) and $Y_{\mathcal{A}}$ is $K^{+}{ }^{-}$bounded,

(iv) $H$ is seq-compact, and there exist a bounded set $B \subset Y$ and a convex cone $C$ such that $K \subset C, H \subset \operatorname{int} C$, and $B \not \subset A+C$ for any $A \in \operatorname{Pr}_{2}(\mathcal{A})$.

Then, for every $(x, A) \in \mathcal{A}$ there exists $(\bar{x}, \bar{A}) \in \mathcal{A}$ such that $(\bar{x}, \bar{A}) \preceq_{H}(x, A)$ and $\mathcal{A} \ni\left(x^{\prime}, A^{\prime}\right) \preceq_{H}(\bar{x}, \bar{A})$ implies $x^{\prime}=\bar{x}$. Moreover, in case (ii), $(\bar{x}, \bar{A})$ can be taken to be a minimal element wrt $\preceq_{H, z^{*}}$ such that $(\bar{x}, \bar{A}) \preceq_{H, z^{*}}(x, A)$.

Proof. It is sufficient to show that condition (C0) is verified. Consider a $\preceq_{H}$-decreasing sequence $\left(\left(x_{n}, A_{n}\right)\right)_{n>1} \subset \mathcal{A}$ with $A_{1} \neq \emptyset$. Using Proposition 4.6 (i), (ii), (iii) or (iv) when (i), (ii), (iii) or (iv) (from our hypothesis) holds, respectively, we obtain that $\left(x_{n}\right)$ is Cauchy. By $(\mathrm{C} 1)$, there exists $(x, A) \in \mathcal{A}$ such that $(x, A) \preceq_{H}\left(x_{n}, A_{n}\right)$ for $n \geq 1$. Hence (C0) holds; the conclusion follows using Theorem 5.1 .

With a similar proof to that of Theorems 5.8 we obtain the next result.

Theorem 5.9 Let $\left(\mathcal{A}, \preceq_{H}\right)$ verify $(\mathrm{C} 1)$ wrt $\preceq_{H}$. Assume that $\left(x_{0}, A_{0}\right) \in \mathcal{A}$ and $\varepsilon>0$ are such that $A_{0} \not \subset A+\varepsilon H+K$ for all $A \in \operatorname{Pr}_{2^{Y}}(\mathcal{A})$. Then there exists $(\bar{x}, \bar{A}) \in \mathcal{A}$ such that $(\bar{x}, \bar{A}) \preceq_{H}\left(x_{0}, A_{0}\right)$, and $\mathcal{A} \ni\left(x^{\prime}, A^{\prime}\right) \preceq_{H}(\bar{x}, \bar{A})$ implies $x^{\prime}=\bar{x}$; moreover, $d\left(\bar{x}, x_{0}\right)<\varepsilon$.

Proof. We apply Theorem 5.1 to

$$
\mathcal{A}_{0}:=\left\{(x, A) \in \mathcal{A} \mid(x, A) \preceq_{H}\left(x_{0}, A_{0}\right)\right\}
$$

preordered by $\preceq_{H}$. For this consider $\left(\left(x_{n}, A_{n}\right)\right)_{n>1} \subset \mathcal{A}_{0}$ a $\preceq_{H^{-}}$decreasing sequence. Then, setting $\left(x_{n}^{\prime}, A_{n}^{\prime}\right):=\left(x_{n-1}, A_{n-1}\right)\left(\in \mathcal{A}_{0}\right)$ for $n \geq 1$, the sequence $\left(\left(x_{n}^{\prime}, A_{n}^{\prime}\right)\right)_{n \geq 1} \subset \mathcal{A}_{0}$ is still $\preceq_{H}$-decreasing. Since $A_{0}=A_{1}^{\prime} \not \subset A_{n}^{\prime}+\varepsilon H+K=A_{n-1}+\varepsilon H+K$ for $n \geq 1$, by Proposition 4.6) (v) we obtain that $\left(x_{n}\right)_{n \geq 1}$ is Cauchy. By $(\mathrm{C} 1),\left(\left(x_{n}, A_{n}\right)\right)_{n>1}$ is minorized in $\mathcal{A}$, and so in $\mathcal{A}_{0}$, too. Hence $(\mathrm{C} 0)$ holds. Applying Theorem 5.1 to $\left(x_{0}, A_{0}\right)\left(\in \mathcal{A}_{0}\right)$, we get $(\bar{x}, \bar{A}) \in \mathcal{A}_{0}$ such that $(\bar{x}, \bar{A}) \preceq_{H}\left(x_{0}, A_{0}\right)$ and $\mathcal{A}_{0} \ni\left(x^{\prime}, A^{\prime}\right) \preceq_{H}(\bar{x}, \bar{A})$ implies $x^{\prime}=\bar{x}$.

Take $\mathcal{A} \ni\left(x^{\prime}, A^{\prime}\right) \preceq_{H}(\bar{x}, \bar{A})$; hence $\left(x^{\prime}, A^{\prime}\right) \in \mathcal{A}_{0}$, and so $x^{\prime}=\bar{x}$. We have to show that $d\left(\bar{x}, x_{0}\right)<\varepsilon$. In the contrary case, $d\left(\bar{x}, x_{0}\right) \geq \varepsilon$. Taking into account that $(\bar{x}, \bar{A}) \preceq_{H}\left(x_{0}, A_{0}\right)$, we get

$$
A_{0} \subset \bar{A}+d\left(\bar{x}, x_{0}\right) H+K \subset \bar{A}+\varepsilon H+\left[d\left(\bar{x}, x_{0}\right)-\varepsilon\right] H+K \subset \bar{A}+\varepsilon H+K,
$$

contradicting the hypothesis $A_{0} \not \subset \bar{A}+\varepsilon H+K$. The proof is complete. 
Of course, the conclusions of Theorems 5.8 and 5.9 remain valid if $(X, d)$ is complete and $\left(\mathcal{A}, \preceq_{H}\right)$ satisfies condition (C'1) instead of $(\mathrm{C} 1)$. Observe that condition (C'1) is introduced in [9, Cor. 35] for $d$ replaced by a regular premetric $\varphi: X \times X \rightarrow \mathbb{R}_{+}$in the definition of $\preceq_{H}$; for $\varphi=d$, [9, Cor. 35] follows from Theorem 5.8 (i) because (A2) implies that $0 \notin \mathrm{cl}_{\text {seq }}\left(K+K^{0}\right)$ [see Lemma 4.4 (i)]. Note that the conclusion of [27, Th. 4.1] is valid only for that $\gamma>0$ appearing in condition (Q3). In this case [27, Th. 4.1] follows from Theorems 5.8 for $H \subset D \backslash(-D)$ instead of $0 \notin \operatorname{vcl}(H+K)$ taking $\mathcal{A}:=\{(x,\{f(x)\}) \mid x \in X\}$; moreover, conclusion (b) implies the more usual estimate $d\left(x_{0}, \hat{x}\right)<\varepsilon / \gamma$. Here, as used in [27], for $A \subset Y$,

$$
\operatorname{vcl} A=\left\{y \in Y \mid \exists v \in Y, \exists\left(\lambda_{n}\right) \subset \mathbb{R}_{+} \text {with } \lambda_{n} \rightarrow 0, \forall n \in \mathbb{N}: y+\lambda_{n} v \in A\right\} .
$$

Theorem 5.3 of the recent paper [28] also follows from Theorem [5.9 applied with $X$ replaced by $\left.S\left(x_{0}\right)\right)$ without using the condition $0 \notin \operatorname{vcl}(H+K)$, but just $0 \notin(H+K)$ with a (somewhat) stronger conclusion. Note also that in Theorem 5.9 we do not use any topology on $Y$ (in such a case, as mentioned in the preliminaries, we could furnish $Y$ with the core convex topology).

Indeed, because of (ii) in [28, Thm. 5.3], one needs only to verify (C1): Consider a decreasing sequence $\left(x_{n}, f\left(x_{n}\right)\right)$. Now, condition (ii) implies that $\left(x_{n}\right)$ is Cauchy [use Proposition 4.6 under condition (v)], and condition (i) implies that $\left(x_{n}\right)$ is convergent to some $x \in X$ with $(x, f(x)) \leq\left(x_{n}, f\left(x_{n}\right)\right)$.

The conditions below depend on the (uniformity defined by the) metric $d$, and they do not depend on $H$. Condition (C'2) corresponds to [29, (H2)] and [15, (H2)]; they will be used for getting a version of Theorem 5.9 similar to the classic EVP.

(C'2) $\forall\left(\left(x_{n}, A_{n}\right)\right)_{n \geq 1} \subseteq \mathcal{A}$ with $\left(A_{n}\right)_{n \geq 1} \leq_{K}^{l}$-decreasing and $x_{n} \rightarrow x \in X: \exists A \in 2^{Y}$ such that $(x, A) \in \mathcal{A}$ and $A \leq_{K}^{l} A_{n} \forall n \geq 1$,

(Ca2) $\forall\left(\left(x_{n}, A_{n}\right)\right)_{n \geq 1} \subseteq \mathcal{A}$ with $\left(A_{n}\right)_{n \geq 1} \leq_{K}^{l}$-decreasing and $\left(x_{n}\right)_{n \geq 1}$ Cauchy : $\left(x_{n}\right)_{n \geq 1}$ is convergent.

Remark 5.6 Observe that $(X, d)$ complete implies (Ca2), and (Ca2) implies (Ca1) wrt $\preceq_{H}$. Having a set-valued mapping $\Gamma: X \rightrightarrows Y$, observe that $\mathcal{A}:=\mathcal{A}_{\Gamma}$ verifies condition (C'2) iff for every sequence $\left(x_{n}\right)_{n \geq 1} \subset X$ one has $\Gamma(x) \leq_{K}^{l} \Gamma\left(x_{n}\right)$ for $n \geq 1$ whenever $x_{n} \rightarrow x \in X$ and $\left(\Gamma\left(x_{n}\right)\right)_{n \geq 1}$ is $\leq_{K}^{l}$-decreasing; in such a case $\Gamma$ is called $K$-sequentially lower monotone $(K$ s.l.m. for short) by Qiu [23, Def. 2.1]. Moreover, in this case (Ca2) reduces to the $(\Gamma, K)$-lower completeness of $(X, d)$ as defined in [23, Def. 2.2] when $F=F_{H}$.

In the next result we provide several conditions; each of them together with (C'2) implies (C'1). Recall that the set $A \subset Y$ is closed in the direction $v \in Y$, or $v$-closed, if $y \in A$ whenever $y+\alpha_{n} v \in A$ for $n \geq 1$ and $\mathbb{R} \ni \alpha_{n} \rightarrow 0 ; A$ is lineally closed if $E$ is closed in any direction $v \in Y$, or equivalently $E=\operatorname{vcl} E$.

Recall that our blanket assumption $(\mathrm{H})$ is working, and so $A \neq \emptyset$ for every $A \in \operatorname{Pr}_{2^{Y}}(\mathcal{A})$.

Proposition 5.10 Let $\left(\mathcal{A}, \preceq_{H}\right)$ verify condition (C'2). Then condition (C'1) is verified provided one of the following conditions holds:

(i) $A+\lambda H+K$ is $v$-closed for some $v \in H+K$ and all $\lambda>0$ and all $A \in \operatorname{Pr}_{2^{Y}}(\mathcal{A})$;

(ii) $H$ is bounded and cs-complete, and $A+K$ is seq-closed for every $A \in \operatorname{Pr}_{2^{Y}}(\mathcal{A})$, 
(iii) (a) the topology of $Y$ is generated by the family $\mathcal{Q}$ of seminorms, (b) either $H$ is cs-complete or else $H$ is cs-closed and $(Y, \mathcal{Q})$ is $\ell^{\infty}$-complet $\mathrm{1}^{1},(\mathrm{c})$ for every $q \in \mathcal{Q}$ there exists $z_{q}^{*} \in K^{+}$such that $q(h) \leq z_{q}^{*}(h)$ for every $h \in H$, and (d) for each $A \in \operatorname{Pr}_{2^{Y}}(\mathcal{A}), A$ is $K^{+}$-bounded and $A+K$ is seq-closed.

(iv) $Y$ is a reflexive Banach space, $H$ is convex and closed, $A$ is $K$-closed and quasi $K$-bounded for every $A \in \operatorname{Pr}_{2^{Y}}(\mathcal{A})$, and there exists $\alpha>0$ such that

$$
\|h+k\| \geq \alpha\|h\| \quad \forall h \in H, \forall k \in K .
$$

Proof. Consider $\left(\left(x_{n}, A_{n}\right)\right)_{n>1} \subset \mathcal{A}$ a $\preceq_{H}$-decreasing sequence with $x_{n} \rightarrow x \in X$. Since $\left(A_{n}\right)_{n \geq 1}$ is clearly $\leq_{K}^{l}$-decreasing, by (C'2), we get $A \in 2^{Y}$ such that $(x, A) \in \mathcal{A}$ and $A \leq_{K}^{l} A_{n}$, that is $A_{n} \subset A+K$, for $n \geq 1$. We have to show that $(x, A) \preceq_{H}\left(x_{n}, A_{n}\right)$ for $n \geq 1$. Clearly, using the transitivity of $\preceq_{H}$, it is sufficient to have that $(x, A) \preceq_{H}\left(x_{n}, A_{n}\right)$ for $n \in P$ with $P$ an infinite subset of $\mathbb{N} \backslash\{0\}$. Because $(x, A) \preceq_{H}\left(x_{n}, A_{n}\right)$ if $x_{n}=x$, we may assume that $x_{n} \neq x$ for $n \geq 1$. Moreover, because $x_{n} \rightarrow x$ (passing to a subsequence if necessary) we may assume that $\sum_{n \geq 1} \delta_{n}<\infty$, where $\delta_{n}:=d\left(x_{n}, x_{n+1}\right)(>0)$ for $n \geq 1$. Replacing $\left(x_{n}\right)_{n \geq 1}$ with $\left(x_{n+p-1}\right)_{n \geq 1}$ for $p \geq 1$, it sufficient to show that $A_{1} \subset A+d\left(x_{1}, x\right) H+K$. Set $\mu:=\sum_{l \geq 1} \delta_{l}$, $\mu_{0}:=0$ and $0<\mu_{p}:=\sum_{l=1}^{p} \delta_{l}$ for $p \geq 1$; clearly, $\mu_{p} \rightarrow \mu$ for $p \rightarrow \infty$.

Take $y \in A_{1}$. Because $A_{1} \subset A_{2}+\delta_{1} H+K$, there exist $y_{2} \in A_{2}, h_{1} \in H, k_{1} \in K$ such that $y_{1}:=y=y_{2}+\delta_{1} h_{1}+k_{1}$. Because $A_{2} \subset A_{3}+\delta_{2} H+K$, there exist $y_{3} \in A_{3}, h_{2} \in H, k_{2} \in K$ such that $y_{2}=y_{3}+\delta_{2} h_{2}+k_{2}$. Continuing in this way we get the sequences $\left(y_{p}\right)_{p \geq 1} \subset Y$, $\left(h_{p}\right)_{p \geq 1} \subset H,\left(k_{p}\right)_{p \geq 1} \subset K$ such that $A_{p} \ni y_{p}=y_{p+1}+\delta_{p} h_{p}+k_{p}$ for $p \geq 1$; in particular, $\left(y_{p}\right)_{p \geq 1}$ is $\leq_{K}-$ decreasing. Hence

$$
y=y_{p+1}+\sum_{l=1}^{p} \delta_{l} h_{l}+k_{p}^{\prime}=u_{p}+\sum_{l=1}^{p} \delta_{l} h_{l}=u_{p}^{\prime}+\mu_{p} h_{p}^{\prime},
$$

where $k_{p}^{\prime}:=\sum_{l=1}^{p} k_{l} \in K$ and $u_{p}:=y_{p+1}+k_{p}^{\prime} \in A+K$ because $y_{p+1} \in A_{p+1} \subset A+K$, and $u_{p}^{\prime} \in A+K, h_{p}^{\prime} \in H$; we used the convexity of $H+K$.

Taking into account (16), if $\mu_{p} \geq d\left(x_{1}, x\right)$ for some $p \geq 1$, from the last expression of $y$ in (46) we obtain that $y \in A+d\left(x_{1}, x\right) H+K$. So, we may (and do) assume that $\mu_{p}<d\left(x_{1}, x\right)$ for $p \geq 1$. Consequently $\mu=d\left(x_{1}, x\right)$ and $\mu_{p}=d\left(x_{1}, x_{p+1}\right)$ for $p \geq 1$.

Assume first that (i) holds. From (46) we have that $y=u_{p}^{\prime}+\mu_{p} h_{p}^{\prime}$, and so

$$
y+\alpha_{p} \bar{y}=u_{p}^{\prime}+\mu_{p} h_{p}^{\prime}+\alpha_{p} v \in A+K+\left(\mu_{p}+\alpha_{p}\right) H+K=A+\mu H+K,
$$

for every $p \geq 1$, where $\alpha_{p}:=\mu-\mu_{p}>0$. Since $A+\mu H+K$ is $v$-closed and $\alpha_{p} \rightarrow 0$, we obtain that $y \in A+\mu H+K=A+d\left(x_{1}, x\right) H+K$.

Assume that (ii) holds. Because $H$ is bounded and cs-complete, the series $\sum_{l=1}^{\infty} \delta_{l} h_{l}$ converges to some $\bar{v} \in Y$ and $h:=\mu^{-1} \bar{v} \in H$. Using the second equality in (46), we get

$$
u_{p}=y-\sum_{l=1}^{p} \delta_{l} h_{l} \rightarrow u:=y-\mu h \in \operatorname{cl}_{s e q}(A+K)=A+K,
$$

and so $y \in A+\mu H+K=A+d\left(x_{1}, x\right) H+K$.

Assume now that (iii) holds. Fix $q \in \mathcal{Q}$ and take $z_{q}^{*} \in K^{+}$such that $q(h) \leq z_{q}^{*}(h)$ for $h \in H$. Because $A$ is $K^{+}$-bounded, $\gamma_{q}:=\inf z_{q}^{*}(A)=\inf z_{q}^{*}(A+K) \in \mathbb{R}$. From (46) we get

$$
z_{q}^{*}(y)=z_{q}^{*}\left(u_{p}\right)+\sum_{l=1}^{p} \delta_{l} z_{q}^{*}\left(h_{l}\right) \geq \gamma_{q}+\sum_{l=1}^{p} \delta_{l} q\left(h_{l}\right)=\gamma_{q}+\sum_{l=1}^{p} q\left(\delta_{l} h_{l}\right),
$$

\footnotetext{
${ }^{1}(Y, \mathcal{Q})$ is $\ell^{\infty}$-complete if for every sequence $\left(y_{n}\right)_{n \geq 1} \subset Y$, the series $\sum_{n \geq 1} y_{n}$ is convergent provided $\sum_{n \geq 1} q\left(y_{n}\right)$ is convergent for every $q \in \mathcal{Q}$ (see [22]).
} 
and so $\sum_{l=1}^{p} q\left(\delta_{l} h_{l}\right) \leq z_{q}^{*}(y)-\gamma_{q}$ for all $p \geq 1$. Hence

$$
\sum_{l=1}^{\infty} q\left(\delta_{l} h_{l}\right)<\infty \quad \forall q \in \mathcal{Q}
$$

This implies hat the sequence $\left(\sum_{l=1}^{p} \delta_{l} h_{l}\right)_{p \geq 1}$ is Cauchy; assuming that $H$ is cs-complete, there exists $h \in H$ such that $\sum_{l=1}^{\infty} \delta_{l} h_{l}=\mu h$. Assuming that $(Y, \mathcal{Q})$ is $\ell^{\infty}$, (47) implies that the series $\sum_{l=1}^{\infty} \delta_{l} h_{l}$ converges to some $v \in Y$; assuming more that $H$ is cs-closed, we have again that $h:=\mu^{-1} v \in H$. As in case (ii) above we obtain that $y \in A+d\left(x_{1}, x\right) H+K$.

Finally, assume that (iv) holds. Taking into account (46) and the fact that $A$ is quasi $K-$ bounded, we get also a bounded sequence $\left(b_{p}\right)_{p} \subset Y$ and the sequences $\left(k_{p}^{\prime \prime}\right)_{p \geq 1},\left(k_{p}^{\prime \prime \prime}\right)_{p \geq 1} \subset K$ such that

$$
y=y_{p+1}+\eta_{p} h_{p}^{\prime}+k_{p}^{\prime \prime}=u_{p}^{\prime}+\mu_{p} h_{p}^{\prime}=b_{p}+\eta_{p} h_{p}^{\prime}+k_{p}^{\prime \prime \prime} \quad \forall p \geq 1 .
$$

Using the last expression of $y$ in (48), from (45) we obtain that $\left\|y-b_{p}\right\| \geq \alpha \eta_{p}\left\|h_{p}^{\prime}\right\|$ for $p \geq 1$, and so $\left(h_{p}^{\prime}\right)_{p \geq 1}$ is bounded. Because $Y$ is reflexive and $H$ is (weakly) closed, $\left(h_{p}^{\prime}\right)_{p \geq 1}$ has a subsequence converging weakly to $h \in H$, and so $h \in H_{p}:=\overline{\operatorname{conv}}\left\{h_{l}^{\prime} \mid l \geq p\right\}$ for every $p \geq 1$. Fix some $p_{1} \geq 1$ such that $d\left(x_{p}, x\right)<1$ for every $p \geq p_{1}$. Because $h \in H_{p_{1}}$, there exists $p_{2}>p_{1}$ and $\left(\lambda_{p}^{1}\right)_{p_{1} \leq p<p_{2}} \subseteq \mathbb{R}_{+}$such that $\sum_{p=p_{1}}^{p_{2}-1} \lambda_{p}^{1}=1$ and $\left\|h^{1}-h\right\|<1$, where $h^{1}:=$ $\sum_{p=p_{1}}^{p_{2}-1} \lambda_{p}^{1} h_{p}^{\prime} \in H$. Increasing if necessary $p_{2}$, we may (and do) assume that $d\left(x_{p}, x\right)<1 / 2$ for every $p \geq p_{2}$. Continuing in this way, we find an increasing sequence $\left(p_{l}\right)_{l \geq 1} \subseteq \mathbb{N}^{*}$ such that for each $l \geq 1$ one has $d\left(x_{p}, x\right)<1 / l$ for $p \geq p_{l}$, and there exists $\left(\lambda_{p}^{l}\right)_{p_{l} \leq p<p_{l+1}} \subseteq \mathbb{R}_{+}$ such that $\sum_{p=p_{l}}^{p_{l+1}-1} \lambda_{p}^{l}=1$ and $\left\|h^{l}-h\right\|<1 / l$, where $h^{l}:=\sum_{p=p_{l}}^{p_{l+1}-1} \lambda_{p}^{l} h_{p}^{\prime} \in H$. Because $\eta_{p}=d\left(x_{1}, x_{p+1}\right) \geq d\left(x_{1}, x\right)-d\left(x_{p+1}, x\right)$, we have that $\eta_{p} \geq d\left(x_{1}, x\right)-1 / l$ for every $p \geq p_{l}$. Using the first expression of $y$ in (48) and the monotonicity of $\left(y_{p}\right)_{p \geq 1}$, we get

$$
y \geq_{K} y_{p+1}+\eta_{p} h_{p}^{\prime} \geq_{K} y_{p+1}+\left(d\left(x_{1}, x\right)-1 / l\right) h_{p}^{\prime} \geq y_{p_{l+1}}+\left(d\left(x_{1}, x\right)-1 / l\right) h_{p}^{\prime}
$$

for $p_{l} \leq p<p_{l+1}$. Multiplying by $\lambda_{p}^{l} \geq 0$ and summing up for $p_{l} \leq p<p_{l+1}$ we get $y \geq_{K} y_{p_{l+1}}+\left(d\left(x_{1}, x\right)-1 / l\right) h^{l}$, and so $y-\left(d\left(x_{1}, x\right)-1 / l\right) h^{l} \in A+K$ for $l \geq 1$. Passing to the limit for $l \rightarrow \infty$ we obtain that $y-d\left(x_{1}, x\right) h \in A+K$ because $A+K$ is closed. Hence $y \in A+d\left(x_{1}, x\right) H+K$.

Note that $0 \notin \operatorname{cl}(H+K)$ when condition (iii) (c) in Proposition 5.10 is verified.

In the next result, we provide several conditions ensuring the $v$-closedness of the set $A+\lambda H+K$ appearing in condition (i) of Proposition 5.10 .

Proposition 5.11 Let $A \subset Y$ be nonempty, $v \in Y \backslash\{0\}$, and $\lambda>0$. Then $A+\lambda H+K$ is $v$-closed provided one of the following conditions holds:

(i) $H$ is seq-compact, and $A+K$ is seq-closed;

(ii) $H+K$ is seq-closed, and $A$ is seq-compact;

(iii) $H$ is a singleton, and $A+K$ is $v$-closed;

(iv) $H+K$ is $v$-closed, and $A$ is finite.

Proof. As already observed, any sequentially closed set is $v$-closed.

(i) Clearly, $\lambda H$ is seq-compact, and so, using Lemma $4.4(\mathrm{i}), A+\lambda H+K[=(A+K)+\lambda H]$ is seq-closed.

(ii) Since $\lambda(H+K)=\lambda H+K$ and the first set is seq-closed, we obtain that $A+\lambda H+K$ is seq-closed as in (i). 
(iii) The assertion is obvious.

(iv) Since $H+K$ is $v$-closed, $\lambda H+K[=\lambda(H+K)]$ is $v$-closed, and so $u+\lambda H+K$ is $v$-closed for every $u \in A$. The conclusion follows from the fact that the union of a finite family of $v$-closed sets is $v$-closed.

Remark 5.7 When $(X, d)$ is complete, in Theorems 5.8 and 5.9 one can replace the hypothesis that (C1) holds with (C'2) together with one of the conditions (i)-(iv) from Proposition 5.10. Even more, instead of assuming that $(X, d)$ is complete in the resulting statement one can assume that (Ca2) or even (Ca1) holds. Moreover, when one needs to have the conclusion for a given $\left(x_{0}, A_{0}\right) \in \mathcal{A}$ (instead of any $\left.(x, A) \in \mathcal{A}\right)$, one may replace $\mathcal{A}$ with $\mathcal{A}_{0}:=\left\{(x, A) \in \mathcal{A} \mid(x, A) \preceq_{H}\left(x_{0}, A_{0}\right)\right\}$ in the hypothesis of the respective statement.

We exemplify (partially) Remark 5.7 with the next result.

Theorem 5.12 Let $\left(\mathcal{A}, \preceq_{H}\right)$ verify conditions (C'2) and (Ca1), as well as one of the conditions (i)-(iv) of Proposition [5.10. Assume that $\left(x_{0}, A_{0}\right) \in \mathcal{A}$ and $\varepsilon>0$ are such that $A_{0} \not \subset A+\varepsilon H+K$ for all $A \in \operatorname{Pr}_{2^{Y}}(\mathcal{A})$. Then for every $\lambda>0$ there exists $\left(x_{\lambda}, A_{\lambda}\right) \in \mathcal{A}$ such that (a) $A_{0} \subset A_{\lambda}+\lambda d\left(x_{\lambda}, x_{0}\right) H+K$, (b) $d\left(x_{\lambda}, x_{0}\right)<\varepsilon / \lambda$, (c) $A_{\lambda} \not \subset A+\lambda d\left(x, x_{\lambda}\right) H+K$ for every $(x, A) \in \mathcal{A}$ with $x \neq x_{\lambda}$.

Proof. Set $d^{\prime}:=\lambda d$ with $\lambda>0$. Of course, for $\left(x_{n}\right)_{n \geq 1} \subset X$ and $x \in X$ we have that $\left(x_{n}\right)$ is $d$-Cauchy (resp. $x_{n} \stackrel{d}{\rightarrow} x$ ) if and only if $\left(x_{n}\right)$ is $d^{\prime}$-Cauchy (resp. $x_{n} \stackrel{d^{\prime}}{\rightarrow} x$ ). Moreover, condition (*) (among (i)-(iv)) is verified wrt $d$ iff $(*)$ is verified wrt $d^{\prime}$. By Proposition 5.10, $\mathcal{A}$ verifies (C'1) wrt $d^{\prime}$, and so, by (28), $\mathcal{A}$ verifies (C1) wrt $d^{\prime}$. Applying Theorem 5.9 we get the conclusion.

It is an easy matter to adapt the preceding results for $\mathcal{A}_{\Gamma}$ with $\Gamma: X \rightrightarrows Y$ because, as seen in Remark 5.6, $\mathcal{A}_{\Gamma}$ verifies (C'2) exactly when $\Gamma$ is $K$-s.l.m., $\mathcal{A}_{\Gamma}$ verifies $(\mathrm{Ca} 2)$ wrt $\preceq_{H}$ exactly when $(X, d)$ is $(\Gamma, K)$-lower complete in the sense of [23, Def. 2.2], and $\mathcal{A}_{0}:=$ $\left\{(x, \Gamma(x)) \mid x \in X, \Gamma\left(x_{0}\right) \subset \Gamma(x)+d\left(x, x_{0}\right) H+K\right\}$ verifies (Ca1) wrt $\preceq_{H}$ exactly when $(X, d)$ is $S\left(x_{0}\right)$-dynamically complete in the sense of [25, Def. 3.1]. So, we get the next version of Theorem 5.12 ,

Corollary 5.13 Let $\Gamma: X \rightrightarrows Y$ be $K$-s.l.m., and let $\mathcal{A}_{\Gamma}:=\{(x, \Gamma(x)) \mid x \in X\}$ satisfy (Ca1) wrt $\preceq_{H}$. Assume that $x_{0} \in \operatorname{dom} \Gamma$ and $\varepsilon>0$ are such that $\Gamma\left(x_{0}\right) \not \subset \Gamma(x)+\varepsilon H+K$ for all $x \in X$, and $\mathcal{A}_{\Gamma}$ verifies one of the conditions (i)-(iv) of Proposition 5.10. Then for every $\lambda>0$ there exists $x_{\lambda} \in X$ such that $\Gamma\left(x_{0}\right) \subset \Gamma\left(x_{\lambda}\right)+\lambda d\left(x_{0}, x_{\lambda}\right) H+K, d\left(x_{0}, x_{\lambda}\right)<\varepsilon / \lambda$, and $\Gamma\left(x_{\lambda}\right) \not \subset \Gamma(x)+\lambda d\left(x, x_{\lambda}\right) H+K$ for every $x \in X \backslash\left\{x_{\lambda}\right\}$.

Remark 5.8 Using Corollary 5.13 one can obtain the following results:

(a) [25, Cor. 3.5] for $\Gamma:=f, K:=D$ and $H:=\left\{k_{0}\right\} \subset K \backslash(-K)$, because condition (i) of Proposition 5.10 [via Proposition 5.11 (iii)] holds in this case; moreover, the assumption that $f(X)$ is quasi $D$-bounded is superfluous.

(b) [25, Cor. 3.6] (for $\lambda$ mentioned in the definition of $S\left(x_{0}\right)$ ) for $\Gamma:=f, K:=D$ and $H:=\left\{k_{0}\right\} \subset K \backslash(-K)$ (instead of $k_{0} \in K \backslash(-\operatorname{vcl} K)$ ), because condition (i) of Proposition 5.10 [via Proposition 5.11 (iii)] holds in this case; moreover, the assumption that $f\left(x_{0}\right) \not \subset$ $f(X)+\varepsilon k_{0}+D$ can be replaced by $f\left(x_{0}\right) \not \subset f(x)+\varepsilon k_{0}+D$ for every $x \in X$. 
(c) [27, Th. 4.2] for $\Gamma(x):=\{f(x)\}, K:=D$ and $H \subset K \backslash(-K)$ (instead of $0 \notin \operatorname{vcl}(H+K))$, because condition (i) of Proposition [5.10 [via Proposition [5.11 (iv)] holds in this case.

(d) As shown in its proof, [27, Th. 4.3] follows from [27, Th. 4.2] because $H \subset D \backslash(-D)$ and $H$ being $\sigma\left(Y, D^{+}\right)$-countably compact imply $0 \notin \operatorname{vcl}(H+D)$. In fact, the same proof shows that in the previous implication one may replace $\operatorname{vcl}(H+D)$ by $\operatorname{cl}_{\text {seq }}(H+D)$.

Similar to Corollary 5.13, the next result is a reformulation of Theorem 5.8, for getting its conclusion apply Theorem 5.8 for $\mathcal{A}:=\mathcal{A}_{\Gamma}$, observing that (C'1) holds.

Corollary 5.14 Let $\Gamma: X \rightrightarrows Y$ be $K$-s.l.m., and let $\mathcal{A}_{\Gamma}$ satisfy (Ca1) wrt $\preceq_{H}$. Assume that $\mathcal{A}_{\Gamma}$ verifies one of the conditions (i)-(iv) of Proposition 5.10. Furthermore, suppose that one of the following conditions holds:

(i) either (a) $0 \notin \mathrm{cl}_{\text {seq }}(H+K)$ and $\Gamma(X)$ is quasi $K$-bounded, or $0 \notin \mathrm{cl}(H+K)$ and $\Gamma(X)$ is $K$-bounded;

(ii) there exists $z^{*} \in K^{+}$such that $\inf z^{*}(\Gamma(X))>-\infty$ and $\inf z^{*}(H)>0$, (iii) $H$ satisfies condition (18) and $\Gamma(X)$ is $K^{+}$-bounded,

(iv) $H$ is seq-compact, and there exist a bounded set $B \subset Y$ and a convex cone $C$ such that $K \subset C, H \subset \operatorname{int} C$, and $B \not \subset \Gamma(x)+C$ for any $x \in X$, Then for every $x \in \operatorname{dom} \Gamma$ there exists $\bar{x} \in S(x)$ such that $S(\bar{x})=\{\bar{x}\}$, where $S(u):=\left\{u^{\prime} \in X \mid \Gamma(u) \subseteq \Gamma\left(u^{\prime}\right)+d\left(u, u^{\prime}\right) H+K\right\}$ for $u \in X$.

Remark 5.9 Replacing $d$ by $\gamma d$, Corollary [5.14 (ii) covers [25, Ths. 4.2 and 4.2'] because $f$ is $D$-s.l.m. (hence (C'2) is verified), and conditions (ii) and (i) of Proposition 5.10 are satisfied, respectively; in [25, Ths. 4.2'] one has $\left(\mathrm{B}_{2}^{\prime}\right) \Rightarrow\left(\mathrm{B}_{1}^{\prime}\right)$, and the boundedness of $H$ is not needed. Corollary [5.14 (i) covers also [25, Th. 4.3] because $f$ is $D$-s.l.m. and condition (iii) of Proposition 5.10 is satisfied.

It was observed in [25, p. 921] that $\Gamma$ is $K$-s.l.m. and has $K$-closed values when epi $\Gamma:=$ $\{(x, y) \in X \times Y \mid y \in f(x)+K\}$ is closed. Using Corollary 5.14 (i), this shows that the conclusion of [15, Th. 10.4.9] remains true. Indeed, (i) of Corollary 5.14 is verified; moreover, when condition (i), (ii), or (iii) of [15, Th. 10.4.9] holds, then condition (ii), (iii), or (iv) of Proposition 5.10 is verified, respectively. Of course, even this variant of [15, Th. 10.4.9] can be strengthened replacing the quasi boundedness of $\Gamma(X)$ by the quasi boundedness of each $\Gamma(x)$ with $x \in X$; moreover, condition (ii) of [15, Th. 10.4.9] could be replaced by conditions (iii) (a)-(c) from Proposition 5.10.

Acknowledgement. The work of the first author was part of the research project "Set Optimization via Abstract Convexity" funded by the Free University of Bozen-Bolzano, the work of the second author was funded by CNCS-UEFISCDI (Romania) under grant number PN-III-P4-ID-PCE-2016-0188.

\section{References}

[1] Brézis, H., Browder, F.E.: A general principle on ordered sets in nonlinear functional analysis. Advances in Math. 21(3), 355-364 (1976)

[2] Cârjă, O.: Some methods of nonlinear functional analysis. MatrixRom, Bucharest (2003) 
[3] Dancs, S., Hegedüs, M., Medvegyev, P.: A general ordering and fixed-point principle in complete metric space. Acta Sci. Math. (Szeged) 46(1-4), 381-388 (1983)

[4] Ekeland, I.: Nonconvex minimization problems. Bull. Am. Math. Soc. 1(3), 443-474 (1979)

[5] Göpfert, A., Riahi, H., Tammer, C., Zălinescu, C.: Variational methods in partially ordered spaces. CMS Books in Mathematics/Ouvrages de Mathématiques de la SMC, 17. Springer-Verlag, New York (2003)

[6] Göpfert, A., Tammer, C., Zălinescu, C.: On the vectorial Ekeland's variational principle and minimal points in product spaces. Nonlinear Anal. 39(7), 909-922 (2000)

[7] Ha, T.X.D.: Ekeland's principle for a set-valued map studied with the set optimization approach (2002), manuscript

[8] Ha, T.X.D.: Some variants of the Ekeland variational principle for a set-valued map. J. Optim. Theory Appl. 124(1), 187-206 (2005)

[9] Hamel, A.H.: Variational principles on metric and uniform spaces. Habilitation thesis, Martin-Luther University Halle-Wittenberg (2005)

[10] Hamel, A., Heyde, F., Löhne, A., Rudloff, B., Schrage, C.: Set optimization-a rather short introduction. In: Set Optimization and Applications-The State of the Art, pp. 65-141. Springer (2015)

[11] Hamel, A., Löhne, A.: Minimal set theorems, report no. 11 (2002), Reports of the Institute of Optimization and Stochastics, Martin Luther University Halle-Wittenberg

[12] Hamel, A., Löhne, A.: Minimal element theorems and Ekeland's principle with set relations. J. Nonlinear Convex Anal. 7(1), 19-37 (2006)

[13] Hamel, A.H., Tammer, C.: Minimal elements for product orders. Optimization 57(2), 263-275 (2008)

[14] Holmes, R.B.: Geometric functional analysis and its applications. Springer-Verlag (1975)

[15] Khan, A.A., Tammer, C., Zălinescu, C.: Set-valued optimization. An introduction with applications. Vector Optimization. Springer, Heidelberg (2015)

[16] Köthe, G.: Topological vector spaces I. Springer-Verlag (1969)

[17] Kuroiwa, D., Tanaka, T., Ha, T.X.D.: On cone convexity of set-valued maps. Nonlinear Analysis: Theory, Methods \& Applications 30(3), 1487-1496 (1997)

[18] Lin, L.J., Chuang, C.S.: Existence theorems for variational inclusion problems and the set-valued vector Ekeland variational principle in a complete metric space. Nonlinear Anal. 70(7), 2665-2672 (2009)

[19] Liu, C.G., Ng, K.F.: Ekeland's variational principle for set-valued functions. SIAM J. Optim. 21(1), 41-56 (2011) 
[20] Luc, D.T.: Theory of vector optimization. Lecture Notes in Economics and Mathematical Systems, vol. 319. Springer-Verlag, Berlin (1989)

[21] Pérez Carreras, P., Bonet, J.: Barrelled locally convex spaces. North-Holland, Amsterdam (1987)

[22] Qiu, J.: Local completeness and dual local quasi-completeness. Proc. Am. Math. Soc. 129(5), 1419-1425 (2001)

[23] Qiu, J.H.: On Ha's version of set-valued Ekeland's variational principle. Acta Math. Sin. (Engl. Ser.) 28(4), 717-726 (2012)

[24] Qiu, J.H.: Set-valued quasi-metrics and a general Ekeland's variational principle in vector optimization. SIAM J. Control Optim. 51(2), 1350-1371 (2013)

[25] Qiu, J.H.: A pre-order principle and set-valued Ekeland variational principle. J. Math. Anal. Appl. 419(2), 904-937 (2014)

[26] Qiu, J.H.: A revised pre-order principle and set-valued Ekeland variational principle (2014), arXiv:1405.1522 v1

[27] Qiu, J.H.: A partial order principle and vector variational principle for $\epsilon$-efficient solutions in the sense of Németh (2016), arXiv:1611.03301 v1

[28] Qiu, J.H.: Ekeland variational principles with set-valued objective functions and setvalued perturbations (2017), arXiv:1708.05126

[29] Tammer, C., Zălinescu, C.: Vector variational principles for set-valued functions. Optimization 60(7), 839-857 (2011)

[30] Turinici, M.: Maximality principles and mean value theorems. An. Acad. Brasil. Ciênc. 53(4), 653-655 (1981)

[31] Turinici, M.: Sequential maximality principles. In: Mathematics without boundaries, pp. 515-548. Springer, New York (2014)

[32] Zălinescu, C.: Convex analysis in general vector spaces. World Scientific Publishing Co. Inc., River Edge, NJ (2002) 